\title{
Optimization of Port Emergency Materials Dispatch with Time Windows Constraints under Uncertainties
}

\author{
Bowei Xu $\mathbb{D}^{1},{ }^{1}$ Yuqing Wang $\mathbb{D},^{1}$ and Junjun Li $\mathbb{D}^{2}$ \\ ${ }^{1}$ Institute of Logistics Science \& Engineering, Shanghai Maritime University, Shanghai 201306, China \\ ${ }^{2}$ Merchant Marine College, Shanghai Maritime University, Shanghai 201306, China \\ Correspondence should be addressed to Bowei Xu; xubowei138@126.com
}

Received 31 July 2021; Accepted 22 October 2021; Published 10 November 2021

Academic Editor: Shi Cheng

Copyright (c) 2021 Bowei Xu et al. This is an open access article distributed under the Creative Commons Attribution License, which permits unrestricted use, distribution, and reproduction in any medium, provided the original work is properly cited.

Uncertainties exist and affect the actual port production. For example, at the beginning of 2020, the sudden outbreak of COVID19 seriously affected terminal production and increased the short-term pressure of handling at container terminals. Consequently, a large number of containers were stacked at terminals, and the problem of terminal congestion became more serious. To solve the congestion problem of container terminals and ensure the priority dispatch of emergency materials, this study uses the optimized arrival patterns of external trucks and a priority dispatch strategy for emergency materials to establish a bilevel optimization model for container terminals and proposes a chaotic genetic algorithm based on logistic mapping as a solution. Through numerical experiments, the algorithm proposed in this study was compared with the genetic algorithm and adaptive genetic algorithm. The experimental results show that the model and algorithm proposed in this study can effectively reduce the total cost of containers in a terminal while ensuring the priority dispatch of emergency materials, reducing the overlapping part of the time window, optimizing the arrival mode of external trucks, and reducing the waiting time of external trucks, effectively alleviating the terminal congestion problem.

\section{Introduction}

The outbreak of COVID-19 in early 2020 has had a serious impact on our lives, industrial production, and economic development. It is a challenge not only for medical and health services but also for the transportation of emergency materials. In this epidemic, the emergency materials are the antiepidemic materials and living materials, mainly including protective clothing, disinfectants, and food. Due to the urgency of time, it is necessary to ensure that all links of emergency logistics are carried out efficiently and quickly.

In response to these situations, the transportation department has actively worked to ensure the smooth transportation of emergency materials. Although the domestic epidemic has been effectively controlled, overseas epidemics have become more severe, affecting terminal logistics. This global public health incident has caused the interruption of the normal operation of container terminals and has affected the original planned production operations of vessels. It is necessary to take corresponding emergency measures to ensure the smooth transportation of emergency materials.

In order to minimize the impact of COVID-19 on our lives, industrial production, and economic development, it is necessary to construct the optimized arrival patterns of external trucks and a priority dispatch strategy for emergency materials in container terminal. When emergency occurs in container terminal, it affects normal production operations. The container terminal should make emergency dispatch of materials, personnel, and equipment to ensure the normal operation of the container terminal. In recent years, the Chinese government has paid more attention to the development of emergency logistics at container terminals and has successively issued measures for container terminal emergency logistics. Container terminal emergency logistics has been substantially developed but still needs further optimization.

Considering that COVID-19 has increased the demand for emergency materials, such as protective clothing, 
disinfectants, and food, time window constraint is used to optimize arrival patterns of external trucks. The arrival patterns of external trucks refer to avoiding the random arrival of external trucks at the container terminal and planning the arrival time of external trucks by using the constraints such as vessel-related time windows; this optimized pattern can alleviate terminal congestion. Then, in response to the need for emergency materials under the outbreak of COVID-19, this article proposes implementing the priority dispatch strategy for emergency materials in container terminals to ensure that emergency materials reach the destinations as soon as possible. The priority dispatching strategy for emergency materials refers to scheduling according to the priority of materials transported by external trucks. We construct a bilevel optimization model for the priority dispatch of emergency materials in container terminals, use the chaotic genetic algorithm based on logistic mapping to solve the problem, and compare the results with the genetic algorithm and the adaptive genetic algorithm to obtain the best plan for priority dispatch of emergency materials.

This study is organized as follows. The literature review on container terminal congestion and emergency logistics at maritime container terminals is discussed in Section 2. The problem description and optimization model are established in Section 3. The solution approach is proposed in Sections 4. Numerical experiments and the results analysis are presented in Section 5. Conclusions and further directions are presented in the last section.

\section{Literature Review}

In response to COVID-19, it is necessary to consider avoiding terminal congestion due to the centralized arrival of the external trucks and giving priority to the external trucks with the task of emergency materials. Therefore, the literature review is mainly organized from two parts: container terminal congestion and emergency logistics.

\subsection{Related Research on Container Terminal Congestion.} Terminal congestion has become a hot topic around the enterprises and academic circles in recent years. Many studies have put forward methods and measures to solve terminal congestion, such as the automated guided vehicle (AGV) route planning, truck appointment system, congestion charging, and some other solutions.

Yang et al. [1] considered the integrated scheduling of quay cranes and AGVs and constructed a bilevel programming model. $\mathrm{Ng}$ [2] replaced the individual limit on the number of deployable ships by examining the method of ship deployment in container ports when there is a time window. Nossack and Pesch [3] solved the truck scheduling problem in intermodal container transportation, and the trucks must be routed and scheduled under the hard time window restrictions imposed by customers and terminals to minimize the total running time of trucks. Shiri and Huynh [4] pointed out that the intermodal terminal requires trucks to make an appointment, and each truck must load and unload containers at the specified time window. The results show that the integrated model can find the best solution. The previously mentioned researches use time windows to solve the problems of integrated terminal dispatch and transportation dispatch.

Some scholars further apply the time window constraint to solve the problem of terminal congestion. For example, Chen et al. [5] proposed a method called "time window dependent on ships" to control the arrival of external trucks. Ma et al. [6] established a two-stage queuing model describing the queuing process of vehicles at gates and yards and established a reservation system based on ship-related time windows. To solve the congestion problem at the gate of the terminal, Yi et al. [7] proposed an appointment scheduling method, which considered the truck cost, the number of appointments allowed in each time window, and the number of trucks available in each time window. Chen and Jiang [8] proposed a time window management solution based on the truck-ship service relationship. Assadipour et al. [9] considered the specific available time window of each crane to minimize the container storage time in the terminal. Although the previously mentioned documents use time windows to solve the problem of terminal congestion, they are all the problems of landside congestion. On this basis, this study takes into account the cooperative planning of landside and seaside to solve the problems of container stacking, busy production, and terminal congestion.

2.2. Related Research on Emergency Logistics. Due to the frequent occurrence of unpredictable disasters, emergency logistics scheduling has become more and more important in modern society, and many studies have proposed solutions.

Minas et al. [10] conducted a comprehensive survey of emergency response operation literature in the article. Research helps strengthen the theoretical basis of emergency response operations. The purpose of the study by Memari et al. [11] was to distribute some temporary emergency stations in the entire area through maximum coverage after a disaster occurs. Qi et al. [12] designed and implemented a postearthquake emergency material distribution and transportation decision support system based on geographic information system in order to reduce the losses caused by the earthquake. However, most of the existing researches consider a certain emergency logistics scheduling model, and most of them are based on ideal scenarios. Considering the uncertainty of information, many scholars have further considered the uncertain scenarios and conducted research. $\mathrm{Lu}$ and Luo [13] pointed out in their research that emergencies are full of a lot of uncertain information, so a new emergency transportation model was proposed in the article, which simulated the emergency transportation scene from logistics centre to disaster areas and between disaster areas. Wang et al. [14] established a multiobjective and multiperiod emergency resource allocation model based on the uncertainty and continuity of the natural rescue process; this model realized the effective distribution of rescue materials and the reasonable choice of transportation routes. 
In 2020, the COVID-19 infectious disease sweeps the world, causing huge loss of life and serious economic problems to countries. Therefore, the provision of epidemic prevention and control materials that guarantee the livelihood of the people is a priority for governments of all countries. In response to this epidemic, some scholars have made researches in the field of transportation. Chen et al. [15] reviewed urban public transportation safety issues, traffic control methods, and emergency public transportation planning in their article. On this basis, they proposed the emergency traffic control measures. Liu et al. [16] used the ant colony-taboo hybrid algorithm to establish a medical waste transportation efficiency model between the hospital and the temporary storage station, which solved the problem of medical waste disposal based on the transit temporary storage station to a certain extent. Pacheco and Laguna [17] considered that the rapid increase in the number of COVID-19 cases has caused the vehicle routing problems. The objective function was processed hierarchically. The first objective was to minimize the transportation time of the longest route. The second goal was to minimize the total travel distance.

The genetic algorithm is widely used in the field of transportation. Park et al. [18] proposed a waiting strategy for the vehicle routing problem with simultaneous pickup and delivery and used the genetic algorithm to solve and validate its accuracy. Escobar-Falcón et al. [19] integrated the fuel consumption heterogeneous vehicle routing problem with two-dimensional loading constraints and presented a specialized genetic algorithm to solve the design of routes. Mahpour et al. [20] determined the fitness function and the initial population and finally imposed constraints in the development process of the genetic algorithm (GA) to determine the optimal service time of container port berths. Avdagic et al. [21] focused on use of the genetic algorithm for effectively narrowing the search space.

In summary, regarding the research on the transportation during the spread of COVID-19, the limitations of these studies are that there is no specific scheduling of container terminal operations, and they did not give priority to the transportation management of epidemic prevention and control materials. Therefore, the main contribution of this article is that, considering the urgency of epidemic prevention and control supplies, priority is given to the dispatch of emergency supplies at container terminals, which has important research significance and research value. Moreover, the genetic algorithm is suitable for solving the related problems in the field of transportation, so this study uses the chaotic genetic algorithm based on logistic mapping to solve the problem.

\section{Model}

In this part, we propose a framework of the bilevel optimization model. Considering the problems of terminal congestion and the dispatch of emergency materials under the uncertain situation, as well as the related time window constraints, establish a bilevel optimization model for the priority dispatch of emergency materials. It can alleviate port congestion while realizing the priority dispatch of emergency supplies. The model framework is shown in Figure 1, and the arrival time window of external truck is solved by the upper-level model and input into the lower-level model as the initial value.

3.1. Problem Description. In the upper layers, with the goal of minimizing the total cost of containers in the container terminal, the arrival time window of external trucks is solved according to constraints, such as the arrival time of the vessel and the storage space of the yard. When the terminal operator receives the arrival notification from the arriving vessel, it will consider the time window for container delivery. The starting point depends on the availability of yard capacity, and the ending point depends on the estimated arrival time of vessel. The upper-level model framework is shown in Figure 2.

There may be overlapping parts in the initial time window between different tasks, which may cause terminal congestion; therefore, the initial time window needs to be adjusted. The lower-level model aims to minimize the cost of task adjustment. According to the priority of the task, the higher the task level is, the higher the adjustment cost is to ensure that urgent materials are dispatched first. Obtaining a time window arrival plan for external trucks with the lowest adjustment cost is the optimal plan for the priority dispatch of emergency materials. The framework of the lower-level model is shown in Figure 3.

3.2. Model Assumptions. Before generating the time windows plan, the information of the upcoming vessels has been known; because container vessels are weekly liners and use the weekly arrival mode, the arrival vessels within one week of container terminal are studied. The container handling quantity of each vessel is known, and the container specifications are assumed to be standard containers with 20 feet. The processing speed of each gate is consistent. The service efficiency of each yard crane is consistent, and the energy consumed by each yard crane is the same.

\subsection{Upper-Level Optimization Model}

3.3.1. Parameter Definition. $i$ : vessel, $i=1,2, \ldots, I$;

$t$ : time, $t=1,2, \ldots, T$

$r$ : the hourly interest rate

$g$ : the number of the gates, $g=1,2, \ldots, G$

$Q_{i}$ : the volume of export containers to be loaded onto vessel $i$

$H$ : the gate processing rate

$A$ : the unit value of waiting time per truck per hour

$B$ : the unit cost of fuel consumption per truck per hour

$Y$ : the total yard storage capacity

$A C$ : the hourly yard fee per container

$V C$ : the average value of one container cargo

$E R_{i t}:$ the estimated arrival time of vessel $i$ 


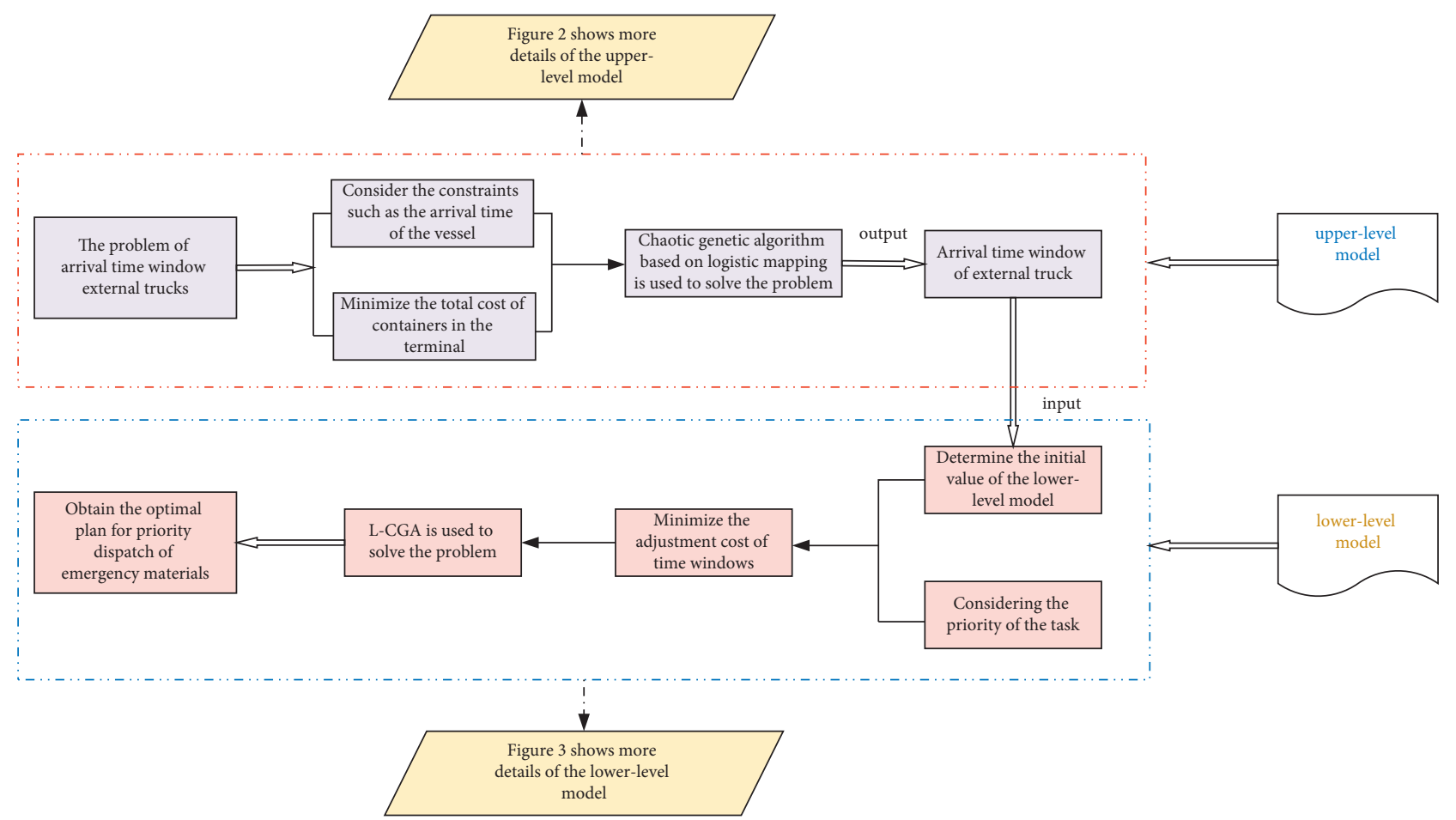

Figure 1: The bilevel optimization model framework.

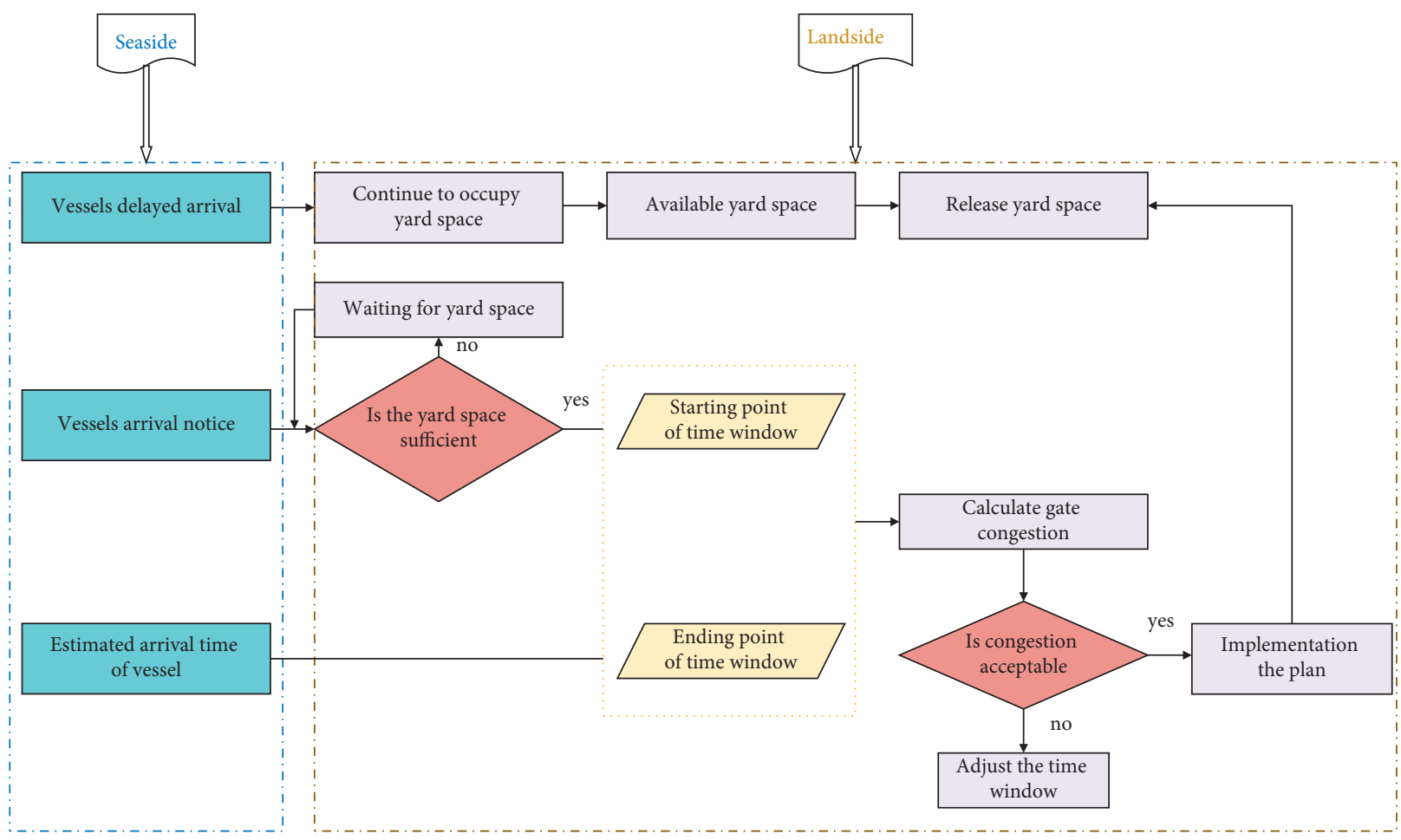

Figure 2: The details of the upper-level model. 


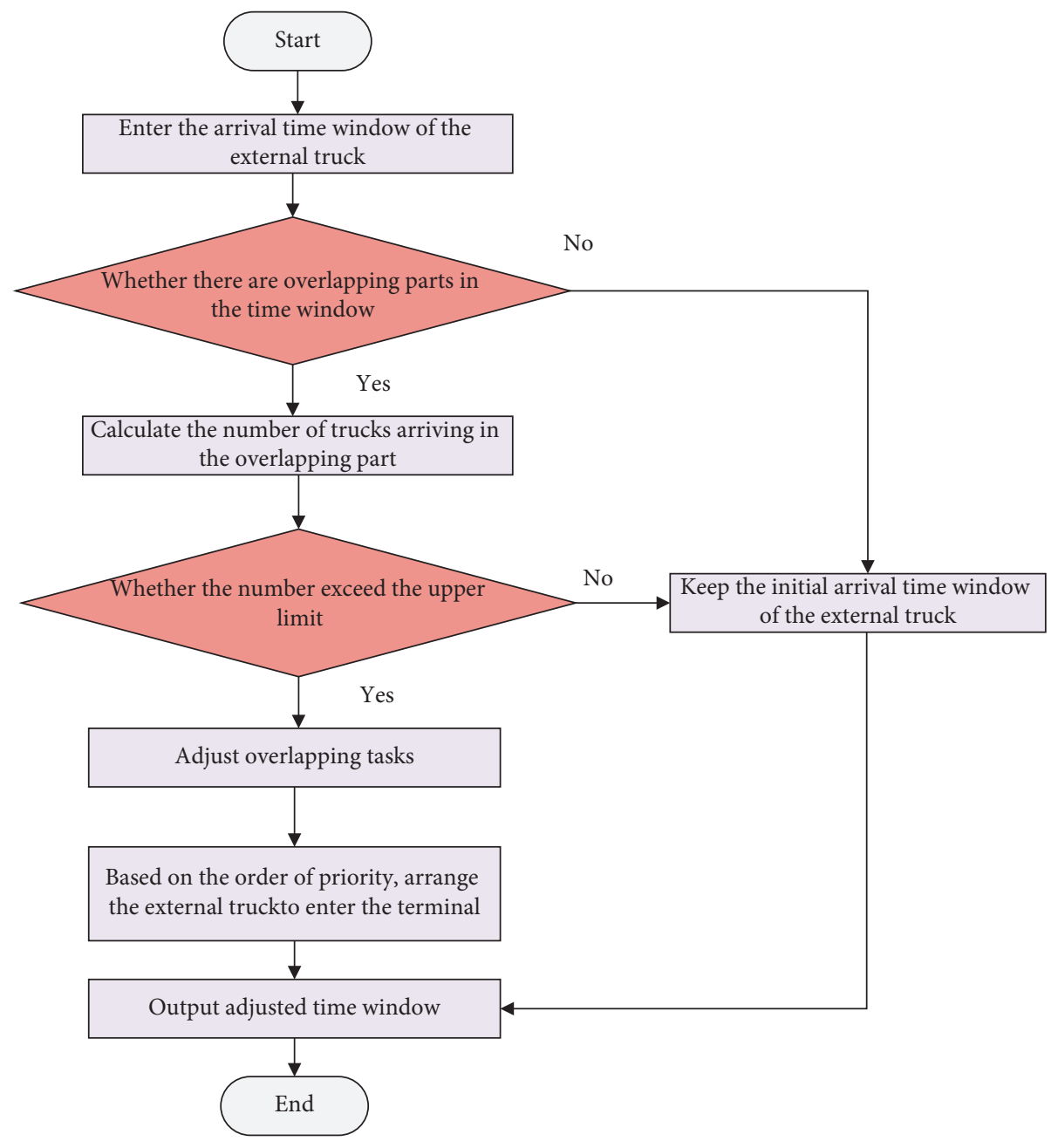

Figure 3: The details of the lower-level model.

$E L_{i t}$ : the estimated departure time of vessel $i$

$T^{l}$ : the length of the time window

$T^{k}$ : the starting point of time window which shall not be earlier than the arrival time hours

$\mathrm{WCO}_{i t}$ : the cost of truck waiting and fuel consumption at time $t$ of vessel $i$

$\mathrm{YCD}_{i t}$ : the yard fee and the storage time cost of cargos at time $t$ for vessel $i$

$P C_{t}$ : the penalty for the insufficient yard space at time $t$ $f_{i t}$ : the number of truck arrivals for vessel $i$ at time $t$ $f_{i t}^{\prime}$ : the number of reallocated truck arrivals for vessel $i$ at time $t$

$n_{t}$ : the queue length

$\bar{\chi}_{t}$ : the average waiting time of the trucks

$D_{i t}$ : the average storage time of the containers arriving at time $t$ for vessel $i$
$T V$ : the value of the storage time per TEU cargo per hour

$\mathrm{O}_{t}$ : the occupied yard space at time $t$

$\lambda_{i t}$ : the number of trucks arriving at the yard at time $t$

$d_{i t}$ : the number of trucks leaving gate $g$ at time $t$

$A Q_{i t}$ : the number of export containers of vessel iat time $t$

$M C$ : the handling charge per container

$N$ : the number of time windows after division

$T L_{i t}=\{1, \quad$ the time windows of time $t$ and time $T-1$ overlap0, others;

$T F_{i t}=\{1$,

the starting point of the time window of the $i$ is at $t 0$, others;

$T A_{i t}$ : starting point of time window

$T B_{i t}$ : ending point of time window 


\subsubsection{Model Establishment.}

$$
\begin{aligned}
\min T C & =W_{1}+W_{2}+W_{3}=\sum_{i} \sum_{t} \mathrm{WCO}_{i t}+\sum_{i} \sum_{t} \mathrm{YCD}_{i t}+\sum_{t} \mathrm{PC}_{t}, \\
W_{1} & =\sum_{i} \sum_{t} \mathrm{WCO}_{i t}=\sum_{i} \sum_{t}\left[(A+B) \times \bar{\chi}_{t} \times f_{i t}^{\prime}\right], \\
W_{2} & =\sum_{i} \sum_{t} \mathrm{YCD}_{i t}=\sum_{i} \sum_{t}\left[(T V+A C) \times D_{i t} \times f_{i t}^{\prime}+N \times M C\right], \\
W_{3} & =\sum_{t} P C_{t}= \begin{cases}999,999, & \text { when } O_{t}>Y, \\
0, & \text { when } O_{t} \leq Y .\end{cases}
\end{aligned}
$$

Regarding all the processes of the container in the terminal, the total cost of container in the terminal is the sum of the cost of external trucks, the cost generated in the yard, and the penalty cost of insufficient space in the yard. The arrival of the external truck should meet the time window constraints related to the vessels, and at the same time, it should ensure that the total cost of the container in the terminal is minimized. The objective function can be expressed by equation (1), where TC means the total cost of container in the terminal.

Equation (2) is the total cost of the external trucks, including the waiting time of the external trucks and the cost of fuel consumption. Equation (3) is the cost of containerized cargo storage time, yard cost, and the cost of manpower and equipment for loading and unloading.

Equation (4) is the penalty cost for insufficient space in the yard. Because of the outbreak, several vessels have not been able to pick up the containers in time, which has led to a backlog of several containers in the yard. In addition, as most countries are in a state of blockade, buyers have begun to cancel orders, leading to an increase in inventory and likely causing congestion. If the yard space is not available at time $t$, an operation halt will happen and all external trucks have to wait at container terminal until some storage space is released. Therefore, a penalty cost is set to avoid the fact that there is no storage space at the yard when the external truck arrives in the terminal, where $O_{t}$ is the occupied yard space at time $t$ and $Y$ is the total yard storage capacity. When the occupied yard space at time $t$ is greater than the total yard storage capacity, a larger penalty cost is set; when the occupied yard space at time $t$ is less than the total yard storage capacity, the penalty cost is 0 .

We have

$$
\begin{gathered}
T B_{i t}-T A_{i t}=T^{l}, \\
T A_{i t}+T^{k} \geq E R_{i t}, \\
T B_{i t}+6 \leq E R_{i t}, \\
T^{l} \geq 6,
\end{gathered}
$$$$
T A_{i t}, T B_{i t} \text { is the integer. }
$$

Each vessel calling at the container terminal is assigned an external truck arrival time window. Equation (5) indicates that the length of the time window for container on the vessel is $T^{l}$. Equation (6) indicates that the starting time of time window should not be earlier than the arrival time of the vessel. Equation (7) indicates that the ending time of the time window should be more than 6 hours earlier than the arrival time of the vessel. Equation (8) indicates that the starting point of each time window should be at least 6 hours earlier than the ending point, which means that the minimum length of the time window is 6 hours. Equation (9) ensures that the starting and ending times for external trucks related to vessel are integers.

$$
f_{i t}= \begin{cases}V_{i_{i}} \times \frac{\int_{c}^{c+\left(i / L_{i}\right)} u^{0.29}(1-u)^{2.25} \mathrm{~d} u}{\int_{0}^{1} u^{0.29}(1-u)^{2.25} \mathrm{~d} u}, & \text { where, } c=\frac{t-1-\sum_{t=1}^{t=T} t * T S_{i t}}{L_{i}}, \quad \text { if, } t \in\left[\sum_{t=1}^{t=T} t * T A_{i t}+1, \sum_{t=1}^{t=T} t * T B_{i t}\right] \forall i, \forall t, \\ 0, & \text { others, } \\ & f_{i t}^{\prime}=f_{i t}+f_{i(t+24 \times 7)}+f_{i(t-24 \times 7)},\end{cases}
$$




$$
\begin{aligned}
n_{t} & =\max \left(n_{t-1}+\sum_{i} f_{i t}^{\prime}-H, 0\right) \\
\bar{\chi}_{t} & =\frac{n_{t}+n_{t-1}}{2 H} \\
\lambda_{i t} & =\sum_{g=1}^{G} d_{i t} \\
O_{t-1} T L_{i t}+\sum_{i} Q_{i} \leq Y & \\
O_{t} & =O_{t-1}+\sum_{i} Q_{i}\left(T F_{i t}-T L_{i t}\right) \\
D_{i t} & =E R_{i t}-t-\bar{\chi}_{t}, \\
T V & =V C \times r \\
A Q_{i t} & =\frac{E_{i}}{\left(T B_{i t}-T A_{i t}+1\right)}, \quad \forall i, t
\end{aligned}
$$

Equations (10)-(13) are constraints at the gate. The truck arrivals can be estimated with equation (10), where $f_{i t}$ is positive only when time $t$ is within the time window $i$; otherwise, $f_{i t}$ is 0 . It is assumed that a terminal has the same vessels calling each week, so, for the vessel operation in the current week, if any workload is allocated to the former or the next week, then the same workload will be allocated back from the next or the former week [10]. Therefore, truck arrivals should be updated with equation (11). Equation (12) is the queue length of external trucks at the gate, and the queue length is measured by the number of trucks. Equation (13) calculates the average waiting time of external trucks at the gate.

Equation (14) calculates the number of external trucks arriving at the yard at time $t$. Equation (15) ensures that the occupied yard space at time $t$ should not exceed the total yard storage capacity in the container terminal. Equation (16) calculates the occupied yard space at time $t$. Equation (17) indicates the average storage time of the containers arriving at time $t$ for vessel $i$. Equation (18) indicates the value of containerized external truck storage time and yard fee. Equation (19) calculates the number of containers related to vessel $i$ arriving at the container terminal gate at time $t$.

\subsection{Lower-Level Optimization Model}

3.4.1. Parameter Definition. $\quad l$ : container, $l=1,2, \ldots, L$

$j$ : external truck, $j=1,2, \ldots, J$

$k$ : the service order of the arrival external truck

$S$ : the capacity of the time window

$U$ : terminal working hours
$M$ : unit time window step

$T R_{i t}$ : the time when the gate actually started to process the task of the external truck

$T A_{i t}^{\prime}$ : starting point of the adjusted time window

$T B_{i t}^{\prime}$ : ending point of the adjusted time window

$Z_{j t}$ : the external truck $j$ arrives at time $t$

$Y_{i t}$ : handling the task of vessel $i$ at time $t$

$C_{\partial}$ : adjustment cost

$\sum_{j \in J} x_{l j}=$

$\{1$, containers $l$ are transported by external truck $j$

$\{0$, others

$\sum_{g \in G} z_{j g}= \begin{cases}1, & \text { external truck } j \text { is served by the gate } g \\ 0, & \text { others }\end{cases}$

$h_{t g}= \begin{cases}1, & \text { the gate } g \text { is working at time } t \\ 0, & \text { others }\end{cases}$

$y_{l j}^{t}= \begin{cases}1, & \text { generate time window adjustment } \\ 0, & \text { others }\end{cases}$

3.4.2. Model Establishment. The goal of the lower-level model is to minimize the adjustment cost of time windows related to the vessels. The higher the priority of the task, the higher the corresponding adjustment cost. Try to adjust the lower priority tasks later to ensure that urgent tasks enter the container terminal first and realize the priority dispatch of urgent materials.

$$
\min \sum_{t \in T} \sum_{j \in J} \sum_{l \in L}\left(y_{l j}^{t} \cdot \frac{\left(T B_{i t}-T A_{i t}\right)-\left(T B_{i t}^{\prime}-T A_{i t}^{\prime}\right)}{N} \cdot C_{\partial}\right),
$$

subject to 


$$
\begin{array}{r}
\partial_{1}+\partial_{2}+\partial_{3}=1, \\
0<\partial_{1}<\partial_{2}<\partial_{3}<1 .
\end{array}
$$

The task level is divided into I, II, and III, and the priority is a specific decimal, which is represented by $\partial_{1}, \partial_{2}$, and $\partial_{3}$. Equations (21) and (22) are used to define the priority of the task, and the task priority remains consistent throughout the container terminal operation. The higher the priority level, the greater the weight, and the sum of their addition is 1 .

$$
\begin{gathered}
N=\frac{U}{M}, \\
T R_{i t}^{\partial 3} \leq T R_{i t}^{\partial 2} \leq T R_{i t}^{\partial 1}, \\
\left(T A_{i t}^{\prime}, T B_{i t}^{\prime}\right) \subset\left(T A_{i t}, T B_{i t}\right), \\
T B_{i t}^{l}-T A_{i t}^{l+1}>0 .
\end{gathered}
$$

Equation (23) is used to calculate the number of time windows after division. Equation (24) is used to ensure that the high-priority external truck enters the terminal earlier than the low-priority external truck. The higher the task level, the earlier the gate processing time. Equation (25) ensures that the adjusted time window is in the initial within the time window. Equation (26) is used to determine whether there is an overlap in the time window.

$$
\begin{aligned}
& T A_{i t} \leq T R_{i t} \leq T B_{i t}, \\
& \sum_{j \in J} x_{l j}=1, \quad l \in L, \\
& \sum_{g \in G} z_{j g}=1, \\
& \sum_{j \in J} y_{g j k} \leq 1, \\
& h_{t g} \leq Z, \quad \forall g \in G, t \in T .
\end{aligned}
$$

Equations (27)-(31) express the constraints related to the gate. Equation (27) guarantees that the time when the gate actually starts processing tasks is within the initial time window of the task. Equation (28) ensures that there is only one task for each external truck. Equation (29) ensures that each external truck is served by only one gate and can only be served once. Equation (30) indicates that one gate can handle at most one task at the same time. Equation (31) ensures that the number of external trucks entering the terminal at the same time does not exceed the total number of gates.

$$
\begin{aligned}
& Z_{j t}=Y_{i t}, \quad \forall i \in I, t \in T, j \in J, \\
& T A_{i t}-T A_{i t}^{\prime} \leq 2 \frac{U}{N} .
\end{aligned}
$$

Equation (32) means that the external trucks serving the same vessel enter the terminal at the same time window.
Equation (33) indicates that the task adjustment range of the external trucks with overlapping time windows is within the interval of the two time windows.

\section{Chaotic Genetic Algorithm Based on Logistic Mapping (L-CGA)}

4.1. The Introduction of Chaotic Genetic Algorithm Based on Logistic Mapping (L-CGA). According to the characteristics of the model, this study introduces the logistic chaotic sequence into the genetic algorithm and generates a chaotic genetic algorithm based on the logistic mapping; it is more compatible with the model in this study.

The difference between the genetic algorithm and chaotic genetic algorithm is that the former adopts the method of random search in the evolution process, whereas the latter uses a chaotic sequence to determine the location of crossover and mutation points, which is the coexistence of randomness and certainty, and is closer to the biological evolution model.

The genetic algorithm has poor local search ability, long solution time, and low search efficiency; it is easy to fall into local convergence and chatter in the optimization process, resulting in premature convergence. The advantage of the chaotic genetic algorithm is that chaotic system is sensitive to initial conditions and has strong ergodicity, which greatly improves the optimization ability of the genetic algorithm.

Introducing chaotic sequences into genetic algorithms can enhance the global search capability by using the randomness and ergodicity of chaos to construct chaotic operators. Chaotic sequence can ensure fast and simple generation and storage, and there is no need to store a long chaotic sequence. Logistic map is a simple one-dimensional map, but it has the characteristics and properties of all chaotic models. It has high efficiency, simple operation, and uniform solution. The expression of logistic chaotic sequence is

$$
\begin{aligned}
x_{n+1} & =f\left(\mu, x_{n}\right)=\mu x_{n}\left(1-x_{n}\right), \\
n & =0,1,2, \ldots, \\
0 & <x_{0}<1, \\
0 & <\mu \leq 4 .
\end{aligned}
$$

The significance of the model is to show accurately the relationship between the fluctuation in the number of biological groups and the changes in the iterations. In the formula, $n$ means the number of iterations, and $x_{n+1}$ and $x_{n}$ are the elements before and after the iteration, respectively. When $\mu=4$, it is completely chaotic.

The specific content of the improvement of the chaotic genetic algorithm based on logistic mapping (L-CGA) is as follows.

4.1.1. Crossover Operation. Based on improved crossover, the logistic chaotic sequence is used to determine the position of the crossover point, and, finally, the crossover item is crossed. Take a random initial value in the range of $(0,1)$, and then, use generate a chaotic value in the range of $(0,1)$, 
Obtain the location of the crossover point from the logistic chaotic sequence

\begin{tabular}{|c|c|c|c|c|c|c|c|c|c|c|c|c|c|c|c|c|c|c|c|c|}
\hline \multirow{3}{*}{$\begin{array}{l}\text { Parent1 } \\
\text { Parent2 }\end{array}$} & TA1 & TA2 & TA3 & TA4 & TA5 & TA6 & TA7 & TA8 & TA9 & TA10 & TB1 & TB2 & TB3 & TB4 & TB5 & TB6 & TB7 & TB8 & TB9 & TB10 \\
\hline & 8 & 33 & 53 & 60 & 73 & 79 & 106 & 106 & 116 & 123 & 21 & 40 & 61 & 66 & 81 & 85 & 112 & 112 & 122 & 138 \\
\hline & 5 & 29 & 44 & 61 & 69 & 76 & 102 & 98 & 108 & 125 & 20 & 37 & 58 & 68 & 78 & 82 & 111 & 106 & 121 & 131 \\
\hline \multirow{2}{*}{$\begin{array}{l}\text { Offspring1 } \\
\text { Offspring2 }\end{array}$} & 8 & 33 & 53 & 60 & 73 & 79 & 106 & 106 & 116 & 123 & 21 & 40 & 58 & 68 & 78 & 82 & 111 & 106 & 121 & 131 \\
\hline & 5 & 29 & 44 & 61 & 69 & 76 & 102 & 98 & 108 & 125 & 20 & 37 & 61 & 66 & 81 & 85 & 112 & 112 & 122 & 138 \\
\hline
\end{tabular}

FIGURE 4: Representation of crossover.

\begin{tabular}{|c|c|c|c|c|c|c|c|c|c|c|c|c|c|c|c|c|c|c|c|c|}
\hline & & & & & & & & & & & & \multicolumn{9}{|c|}{ Mutation point } \\
\hline & TA1 & TA2 & TA3 & TA4 & TA5 & TA6 & TA7 & TA8 & TA9 & \begin{tabular}{|l|} 
TA10 \\
\end{tabular} & TB1 & TB2 & TB3 & TB4 & TB5 & TB6 & TB7 & TB8 & TB9 & TB10 \\
\hline Parent & 8 & 33 & 53 & 60 & 73 & 79 & 106 & 106 & 116 & 123 & 21 & 40 & 61 & 66 & 81 & 85 & 112 & 112 & 122 & 138 \\
\hline & & & & & & & & & & & & & & & & & & & & \\
\hline fspring & 8 & 33 & 53 & 60 & 73 & 79 & 106 & 106 & 112 & 123 & 21 & 40 & 61 & 66 & 81 & 80 & 112 & 112 & 122 & 138 \\
\hline
\end{tabular}

Obtain the location of the mutation point from the logistic chaotic sequence

FiguRE 5: Representation of mutation.

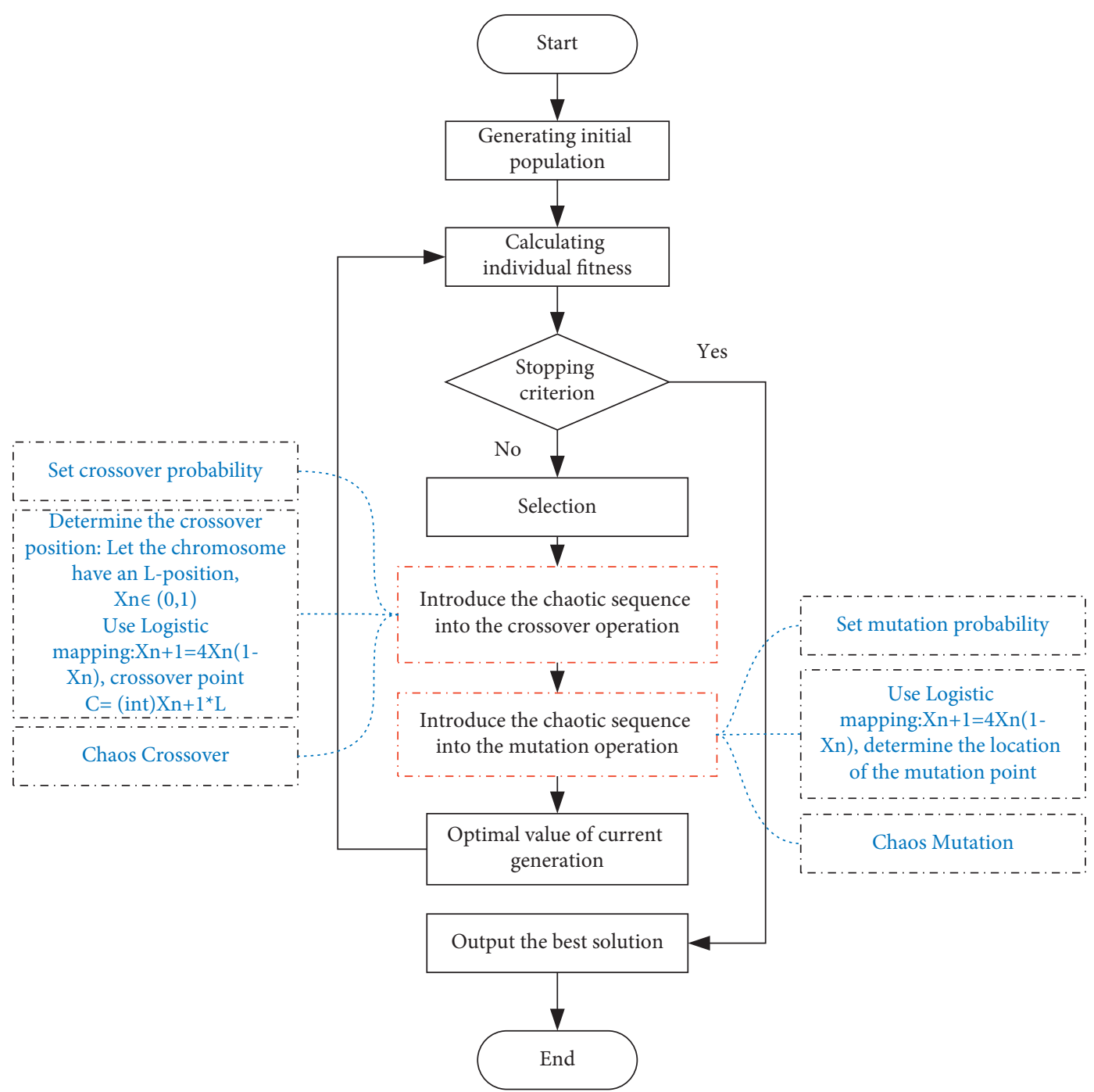

FIGURE 6: Implementation procedure of the chaotic genetic algorithm based on logistic mapping. 


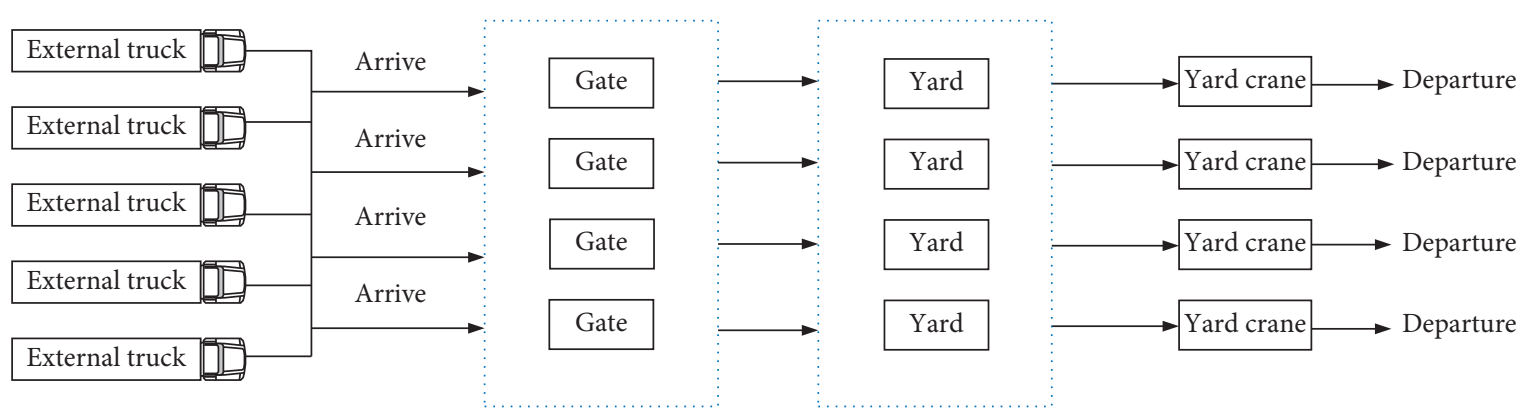

FIgURE 7: Operation process of external trucks.

TABLE 1: Input variables and input values.

\begin{tabular}{lc}
\hline Input variable & Input value \\
\hline Diesel idling fuel consumption & $3.81 / \mathrm{h}$ \\
Price of fuel & $6 \mathrm{yuan} / 1$ \\
Time value of truck/driver waiting & $40 \mathrm{yuan} / \mathrm{h}$ \\
Average value of container cargo & $184,000 \mathrm{yuan} / \mathrm{TEU}$ \\
The rate of interest & $5 \% / \mathrm{year}$ \\
The gate processing rate & $40 \mathrm{trucks} / \mathrm{h}$ \\
The minimum length of time window & $6 \mathrm{~h}$ \\
Number of gate lanes & 4 \\
\hline
\end{tabular}

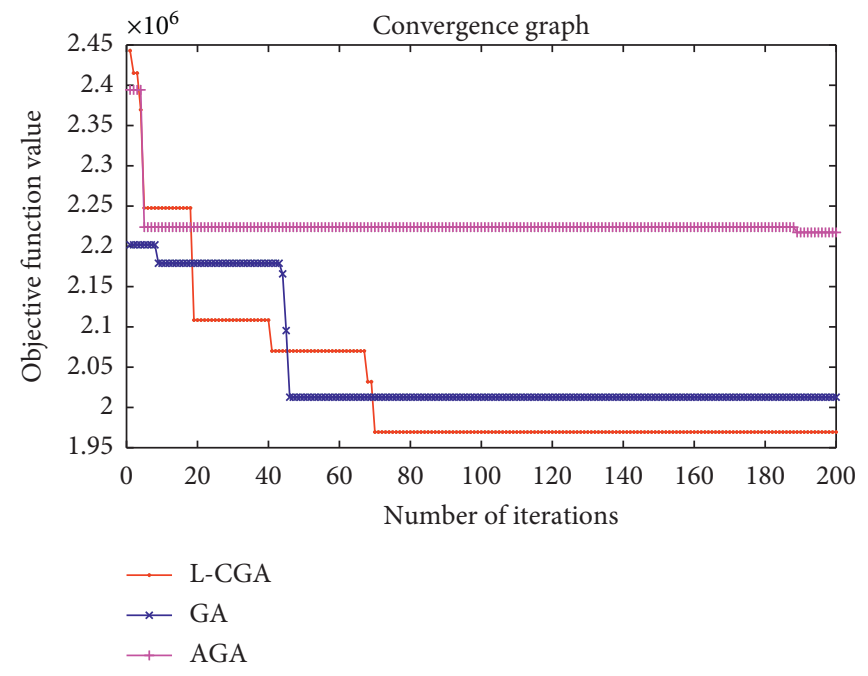

FIgURe 8: Convergence curve.

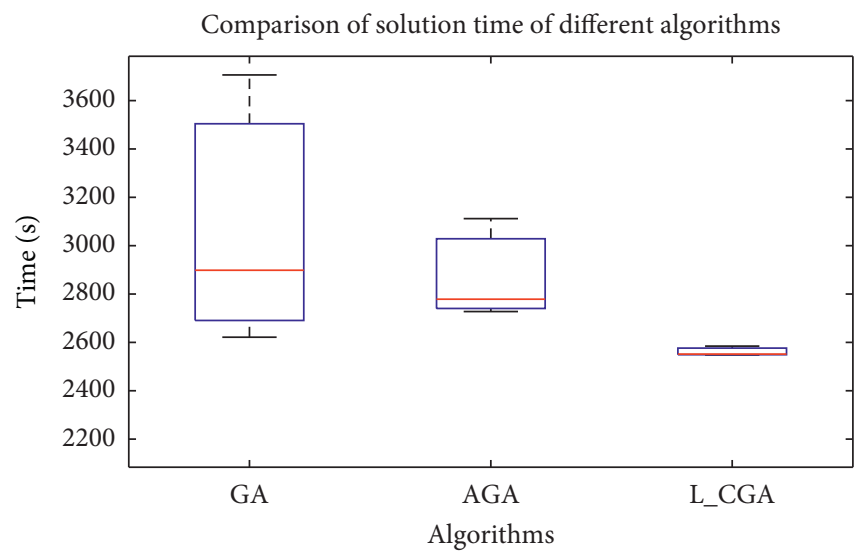

FIGURE 9: Comparison of solution times of different algorithms. 


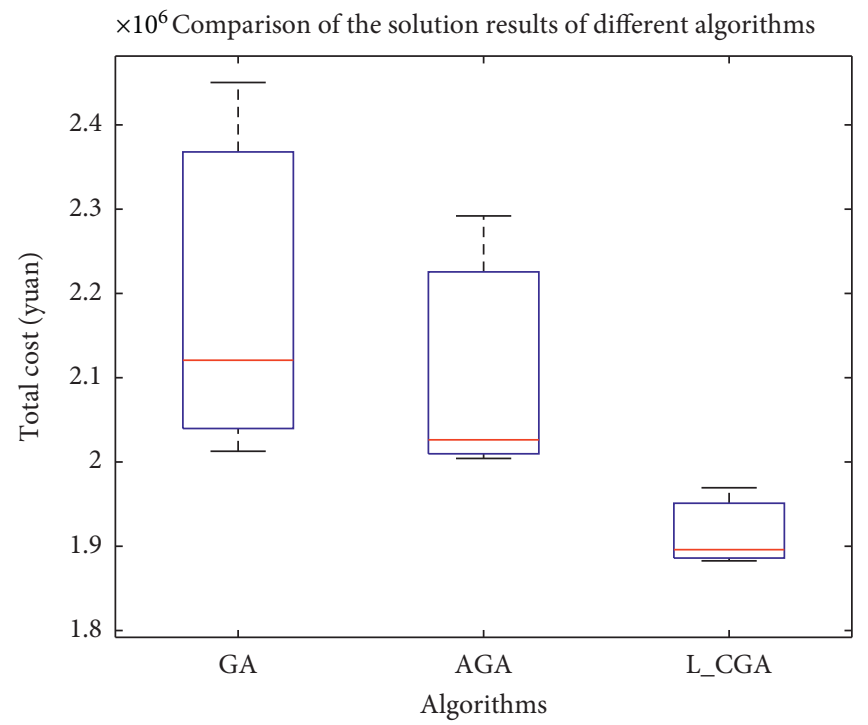

FIgURE 10: Comparison of the solution results of different algorithms.

TABLE 2: Estimated times of arrival and departure of vessels.

\begin{tabular}{|c|c|c|}
\hline Vessel no. & Estimated arrival time & Estimated departure time \\
\hline 1 & Tuesday 4:00 & Tuesday 18:00 \\
\hline 2 & Tuesday 8:00 & Tuesday 23:00 \\
\hline 3 & Tuesday 16:00 & Wednesday 7:00 \\
\hline 4 & Tuesday 22:00 & Wednesday 21:00 \\
\hline 5 & Tuesday 23:00 & Wednesday 8:00 \\
\hline 6 & Wednesday 5:00 & Wednesday 15:00 \\
\hline 7 & Wednesday 9:00 & Wednesday 23:00 \\
\hline 8 & Wednesday 15:00 & Thursday 7:00 \\
\hline 9 & Wednesday 16:00 & Thursday 1:00 \\
\hline 10 & Wednesday 17:00 & Thursday 7:00 \\
\hline 11 & Wednesday 20:00 & Thursday 9:00 \\
\hline 12 & Thursday 1:00 & Thursday 13:00 \\
\hline 13 & Thursday 2:00 & Thursday 18:00 \\
\hline 14 & Thursday 5:00 & Thursday 17:00 \\
\hline 15 & Thursday 13:00 & Friday 0:00 \\
\hline 16 & Thursday $16: 00$ & Friday 2:00 \\
\hline 17 & Thursday 20:00 & Friday $16: 00$ \\
\hline 18 & Thursday 22:00 & Friday 19:00 \\
\hline 19 & Friday 0:00 & Friday 11:00 \\
\hline 20 & Friday 3:00 & Friday 21:00 \\
\hline 21 & Friday 22:00 & Saturday $13: 00$ \\
\hline 22 & Saturday 2:00 & Saturday $16: 00$ \\
\hline 23 & Saturday 8:00 & Saturday 23:00 \\
\hline 24 & Saturday 19:00 & Sunday 11:00 \\
\hline 25 & Saturday 20:00 & Sunday 6:00 \\
\hline 26 & Saturday 21:00 & Sunday 9:00 \\
\hline 27 & Saturday $22: 00$ & Sunday $14: 00$ \\
\hline 28 & Sunday 0:00 & Sunday 9:00 \\
\hline 29 & Sunday 2:00 & Sunday 23:00 \\
\hline 30 & Sunday $12: 00$ & Sunday $22: 00$ \\
\hline
\end{tabular}

save the above chaotic value as the initial value of chaotic iteration for generating the next generation of cross terms, and the specific process is shown in Figure 4. This single- point crossover changes the original solution very little, which can weaken and avoid the optimal chattering problem and improve the accuracy of algorithm convergence. 


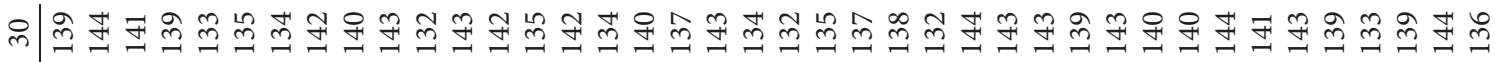

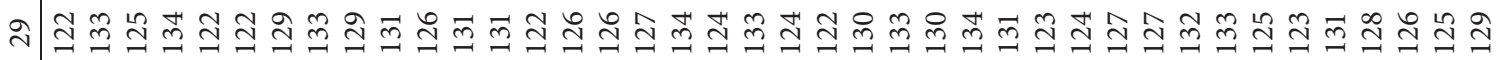

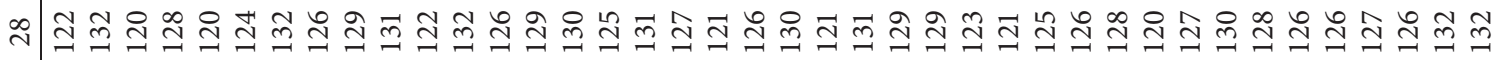

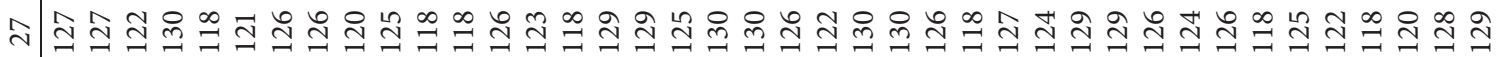

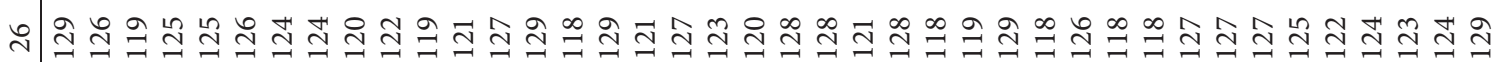

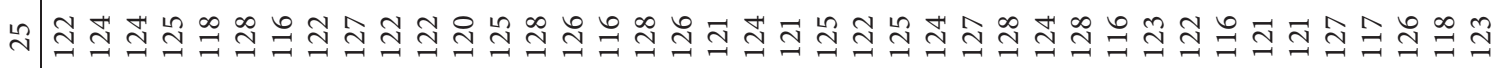

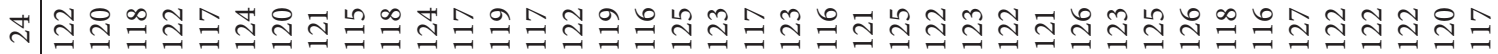

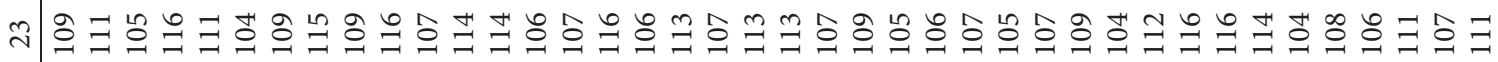

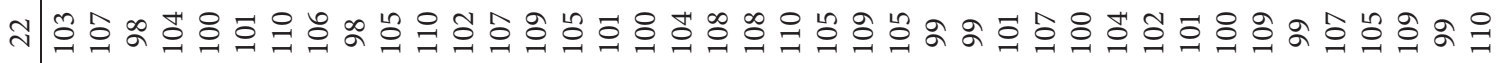

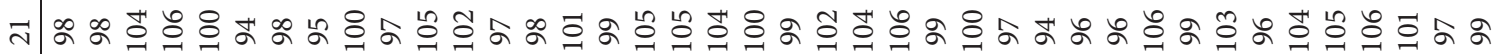

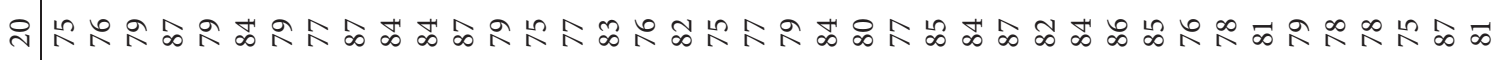

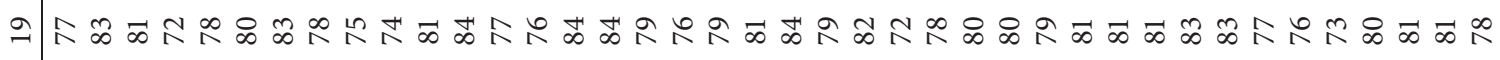

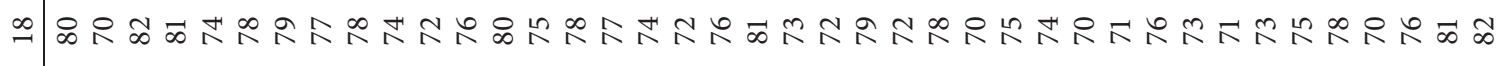

ต H

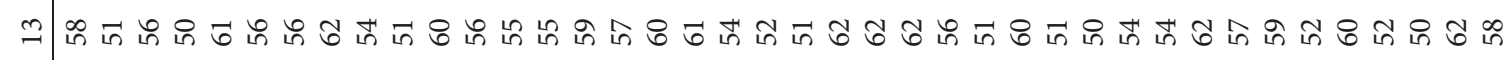

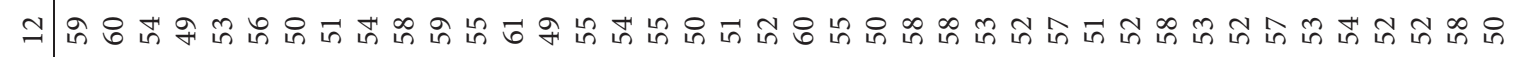

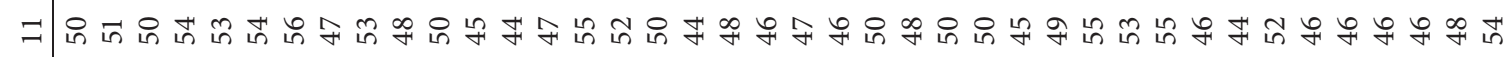

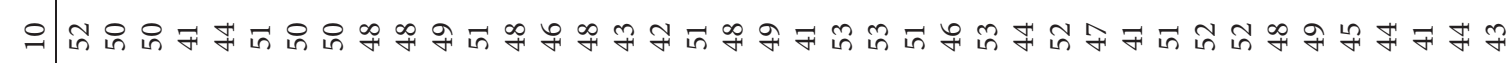

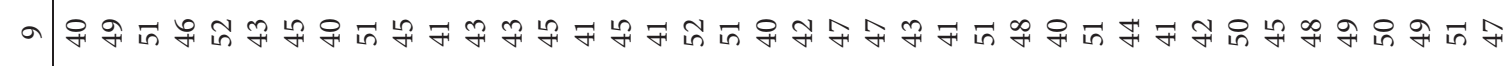

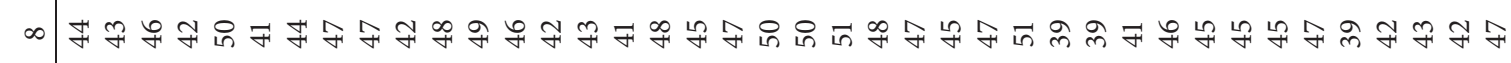

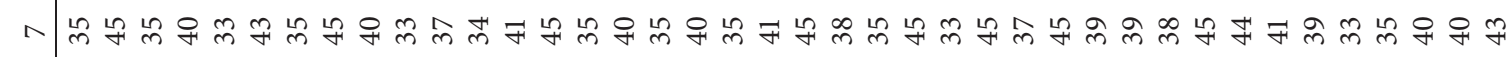
○) ద +

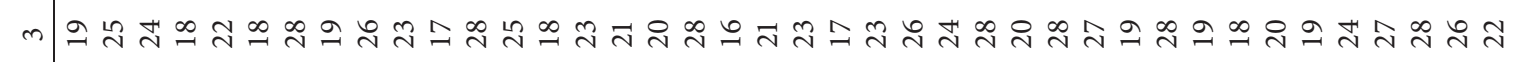
N

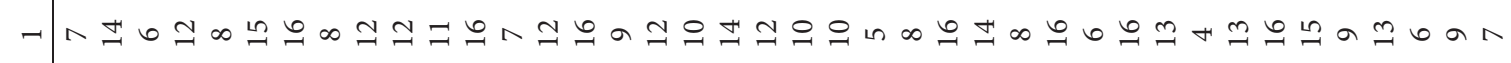

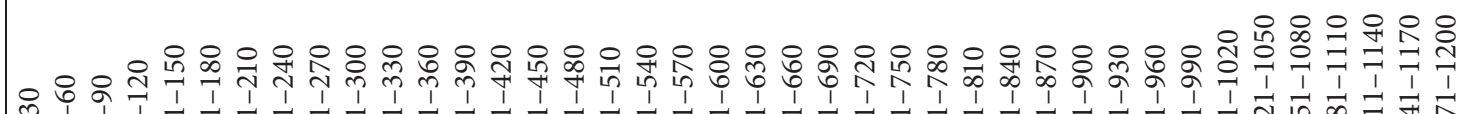

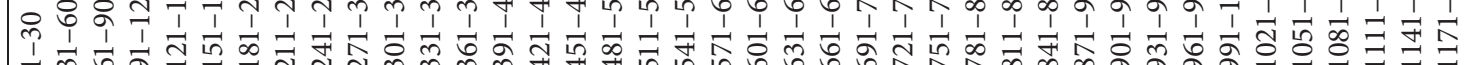

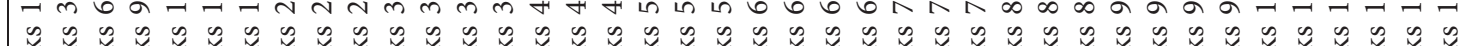

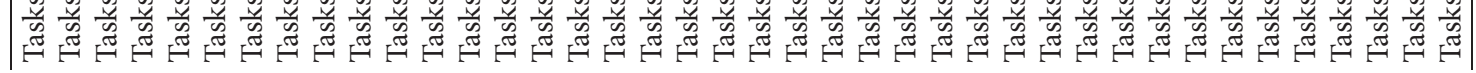




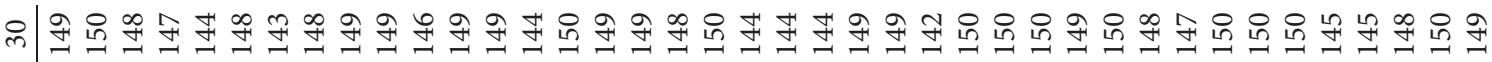

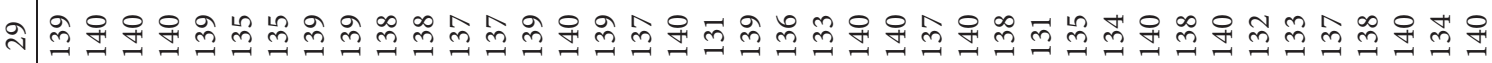

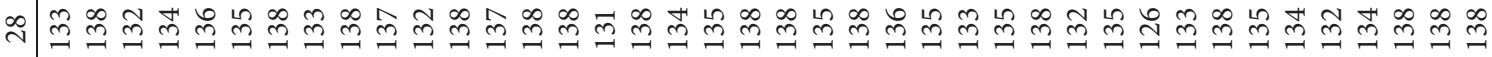

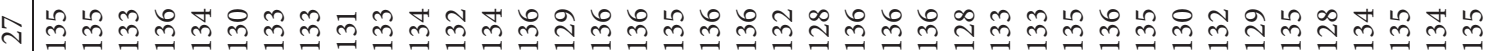

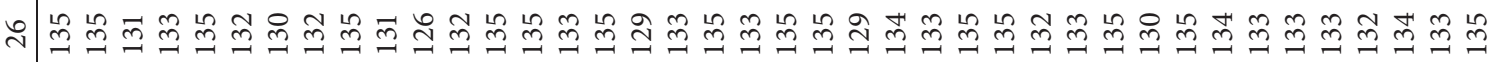

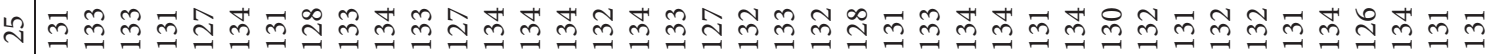

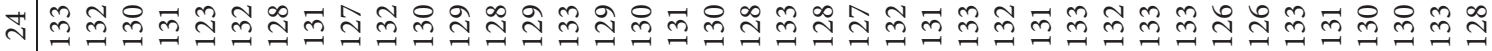

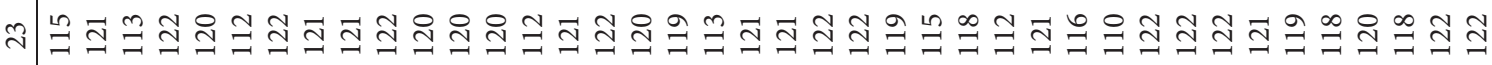

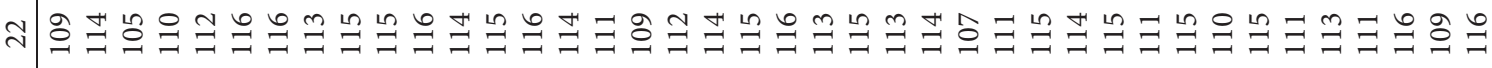

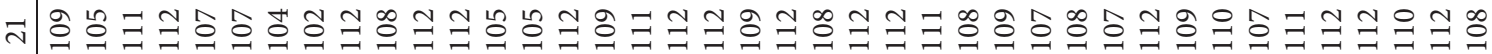

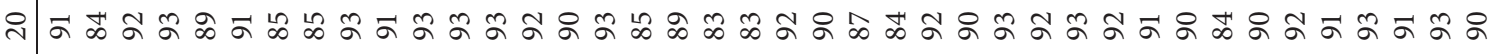
ᄀ|

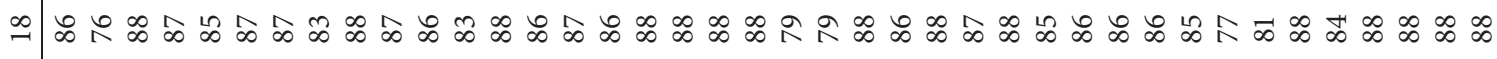
규

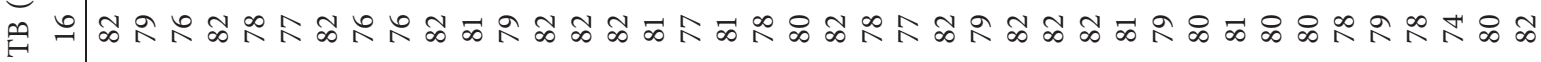

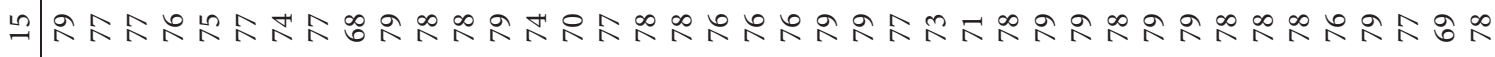

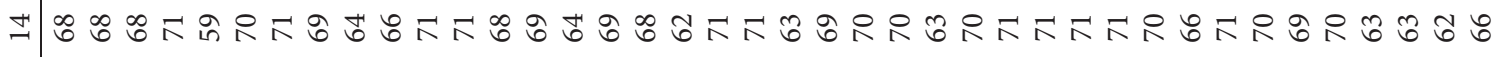
m

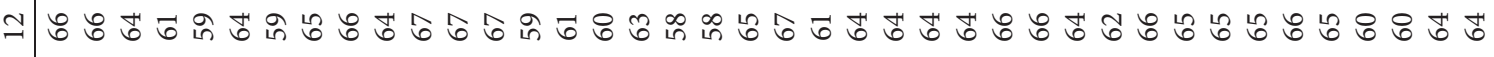

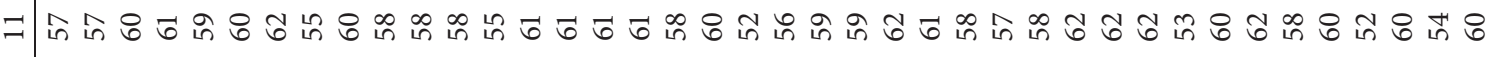

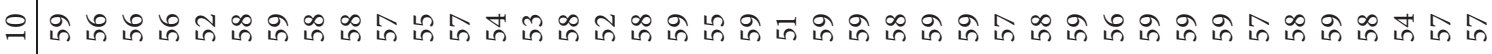

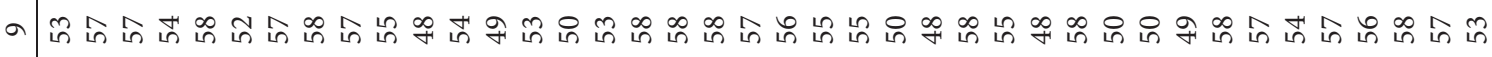

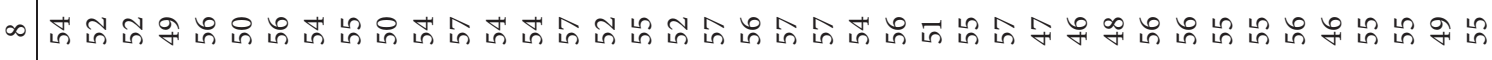

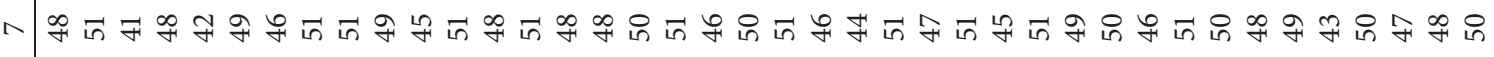

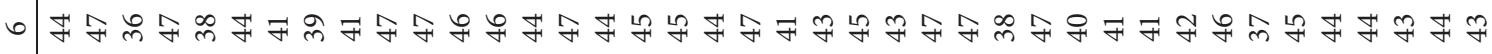

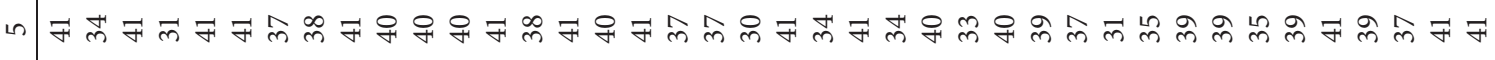
+

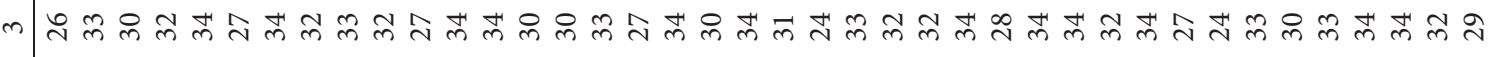
N

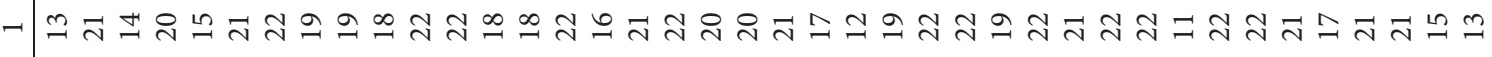

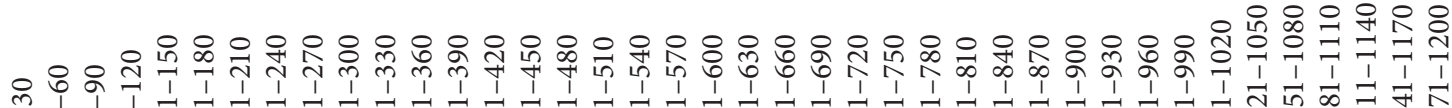

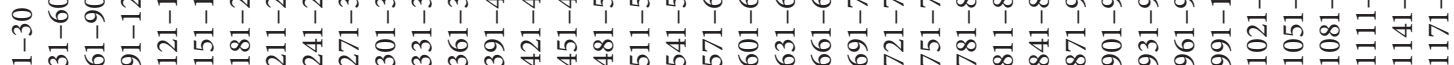

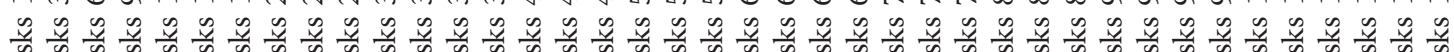

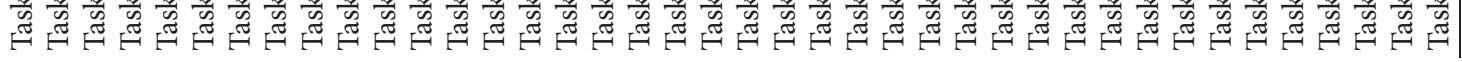




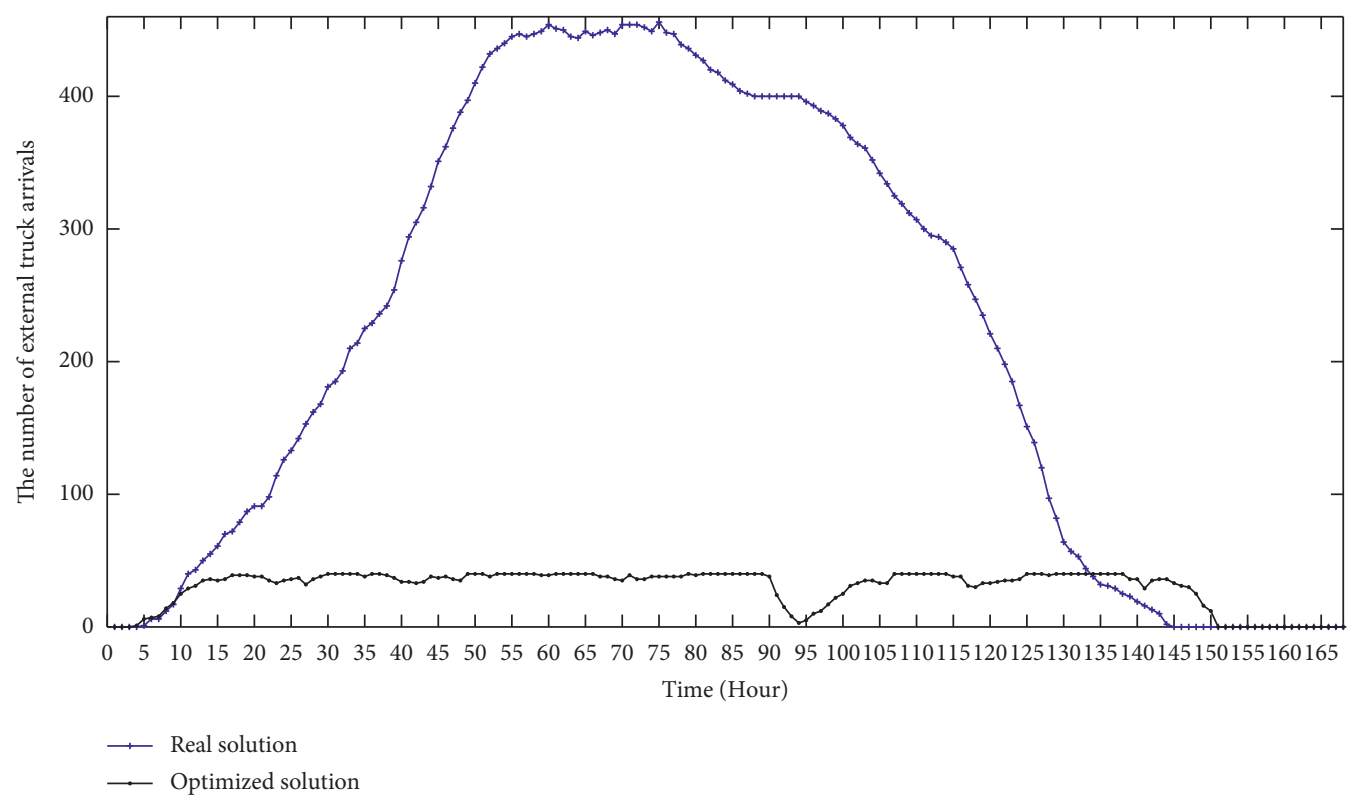

FIgURE 11: The truck arrivals in the real and optimized cases.

4.1.2. Mutation Operation. Mutation is also a means to achieve group diversity and an important guarantee for jumping out of local optimal. The specific mutation operator in this study is designed as follows. According to the given mutation rate, the specific mutation takes the current gene value as the initial value and uses the chaotic sequence to perform an appropriate number of iterations to obtain the new gene value after the mutation, thereby obtaining a new chromosome. The specific process is shown in Figure 5.

\subsection{Implementation Procedure of Chaotic Genetic Algorithm} Based on Logistic Mapping. Figure 6 shows the implementation procedure of the chaotic genetic algorithm based on logistic mapping. In this study, the chaotic genetic algorithm based on logistic mapping (L-CGA) is conducted with the following steps:

(1) Coding method and population initialization: the chromosome is composed of the start and end points of the time window, and each is coded by integer number. The chromosome can be represented as $\left(T A_{1 t}, T A_{2 t}, \ldots, T A_{I t}, T\right.$ $\left.B_{1 t}, T B_{2 t}, \ldots, T B_{I t}\right)$. This study adopts the approach of initializing individuals randomly under the constraints of equations (5)-(9), ensuring that every individual is feasible.

(2) Fitness evaluation: the fitness value is the reciprocal value of the total cost of external trucks in the terminal

(3) Select: to select a parent, a roulette wheel approach is applied. The highest fitness individual of current population is retained to the next generation to ensure that the final outcome of L-CGA is the highest fitness individual of all generations.

$$
P_{i}=\frac{f_{i}}{\sum_{i=1}^{M} f_{i}},
$$

where $f_{i}$ is the individual fitness value and $P_{i}$ is the probability of being selected.

(4) Crossover

(1) Control the frequency of crossover operation and decide on crossover. Use single-point crossover and preset the crossover probability. Any initial value will be used as a criterion, if crossover probability is less than this value, the crossover operation can be performed; otherwise, it will not crossover. Liu et al. [22] pointed out in the study that a large number of experiments show that when crossover probability is set to 0.9 , the performance of the chaotic genetic algorithm is the best.

(2) The logistic chaotic sequence determines the location of the crossover. Consider a long chromosome with $L$ position, randomly select a number in the interval $(0,1)$ as the initial value, and use the logistic model to generate a chaotic sequence in the interval $(0$, $1)$. Use the formula to map the sequence to the chromosome, so that the crossover operation occurs at this position to form a new offspring; only parts of the genes are updated, so the changes are small to avoid optimal chattering.

(5) Mutation operation

(1) Control the frequency of mutation operation, randomly select the mutation probability, and set it in advance. The mutation probability is set as 0.1 [22]. 


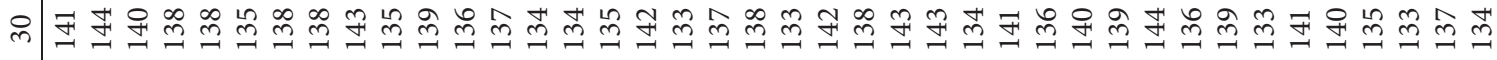

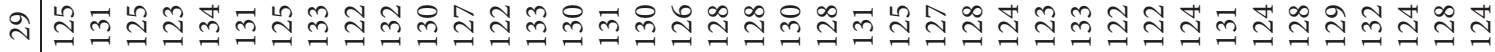

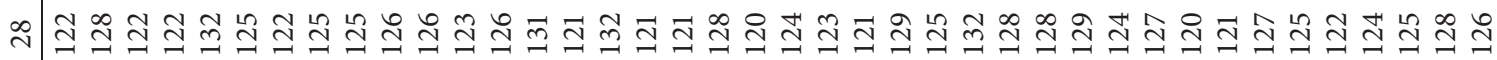

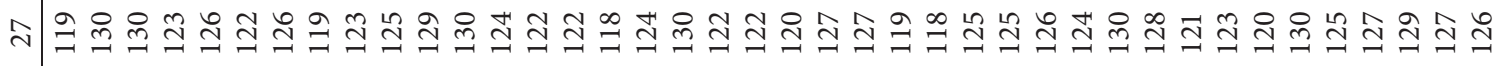
념 น

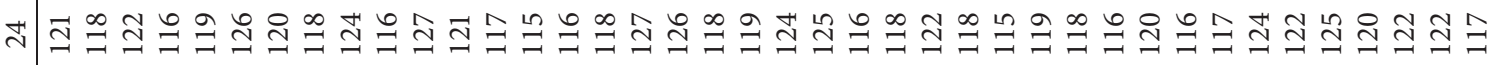

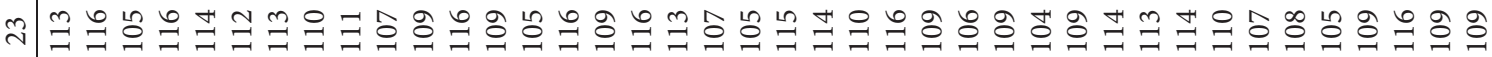

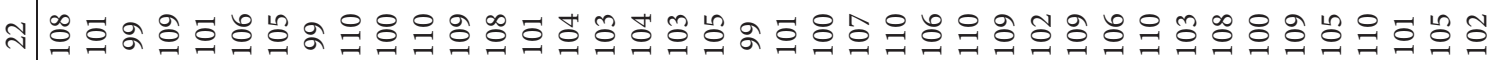

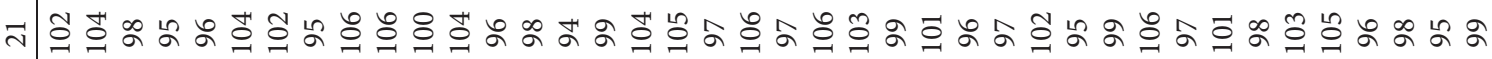

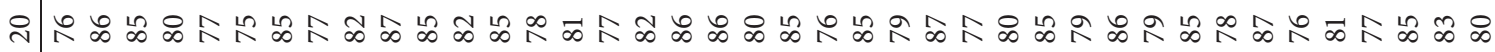

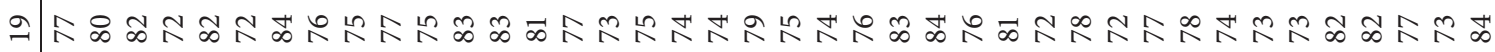

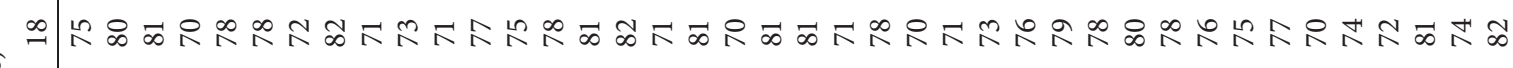

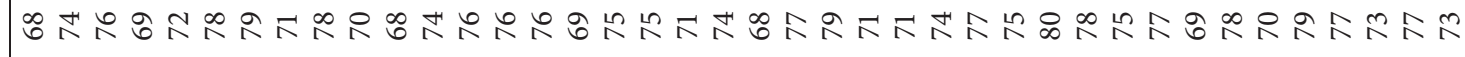

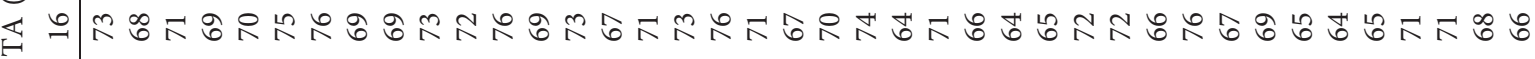

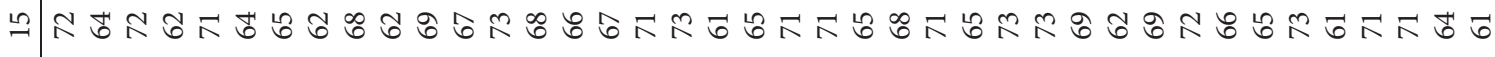

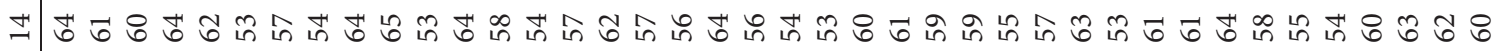

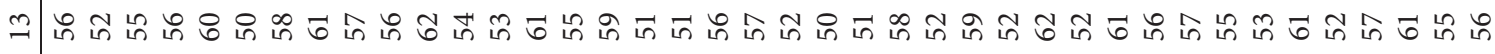

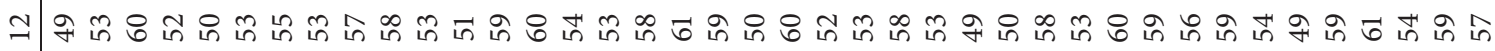

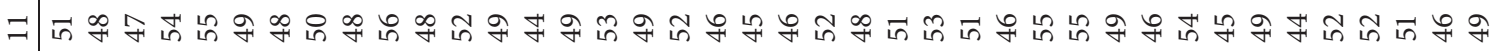

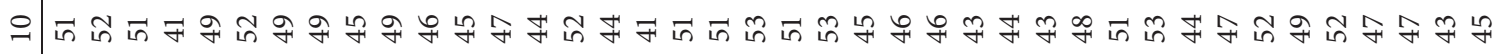

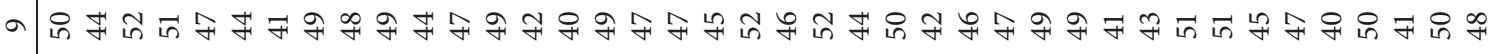

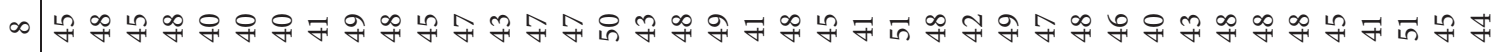

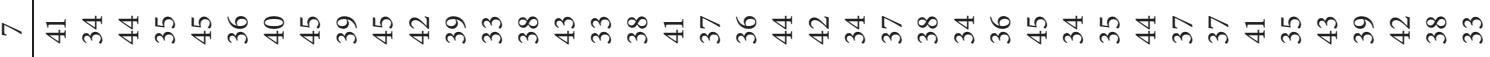

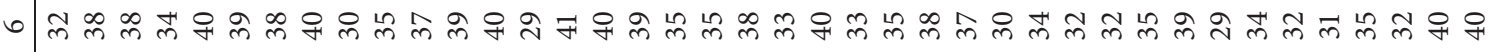

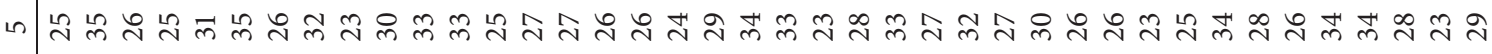

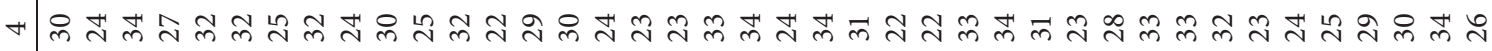

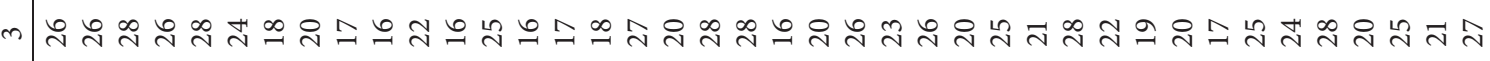

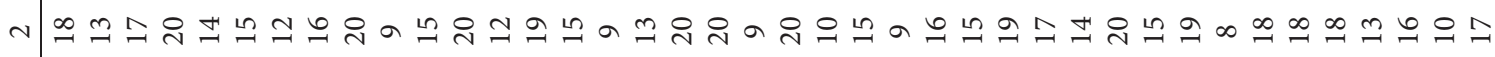

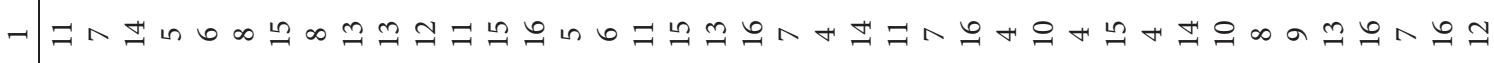

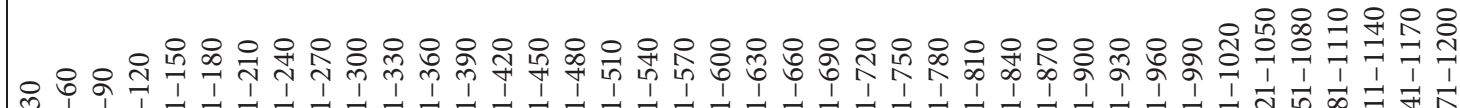

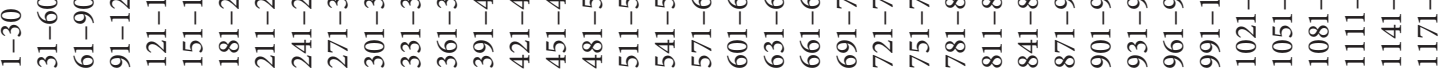
S

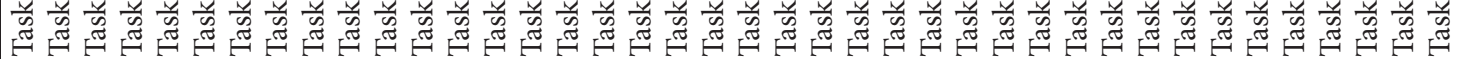




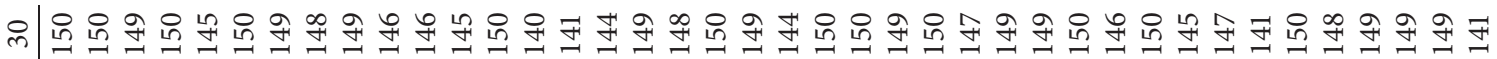

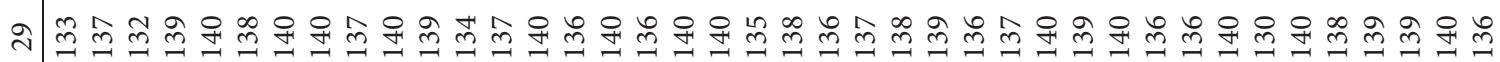

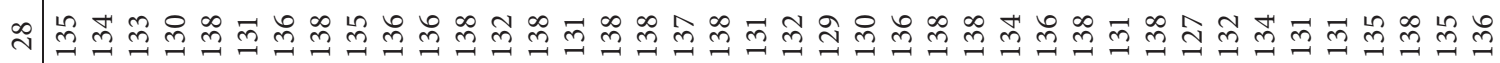

సิ

¿

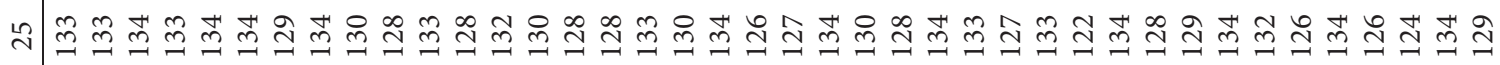

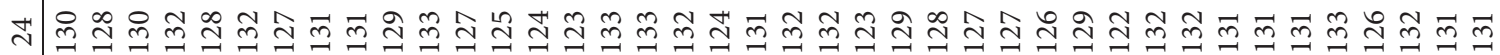

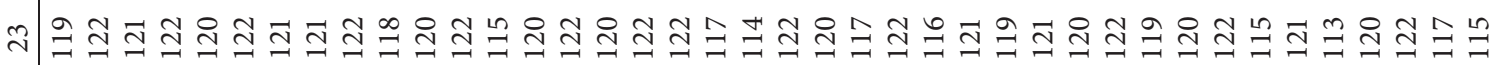

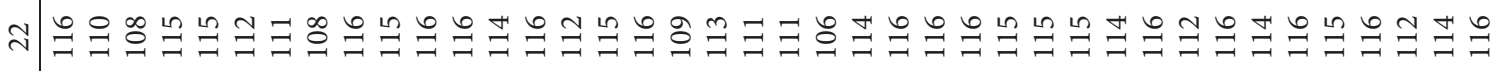

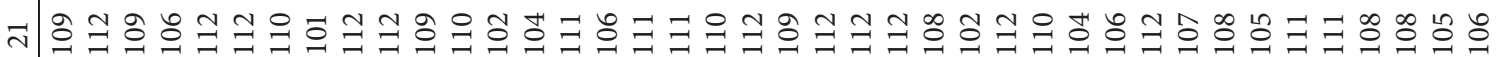

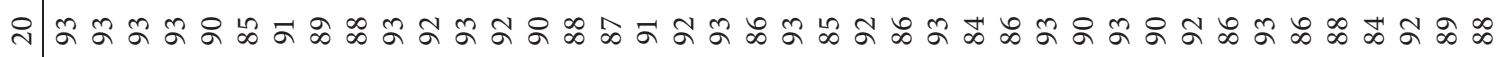

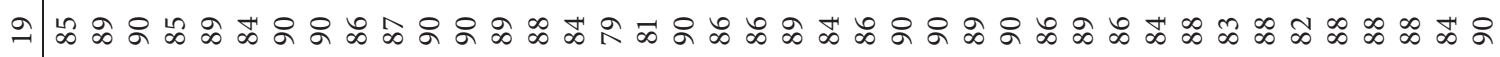

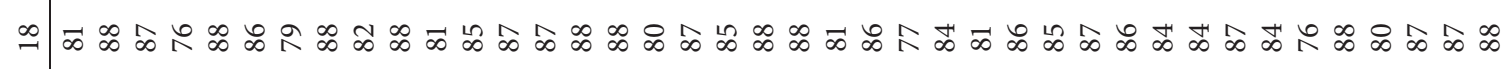

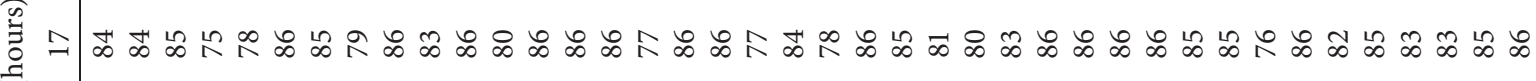

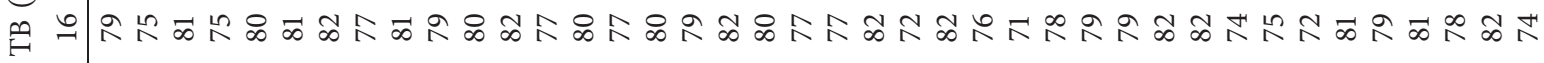

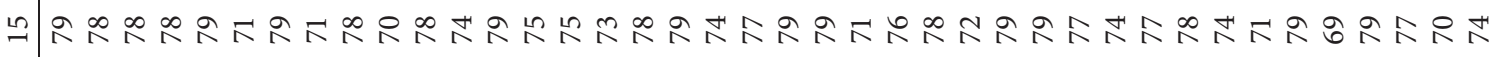

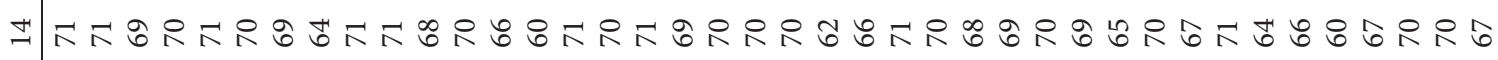

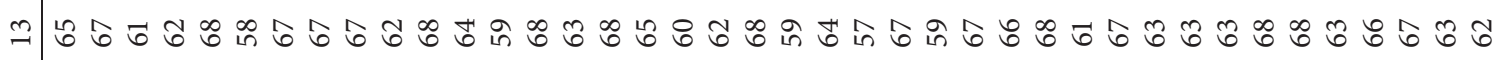
긍

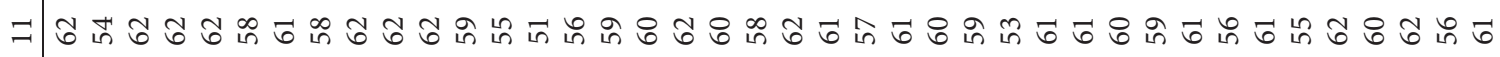

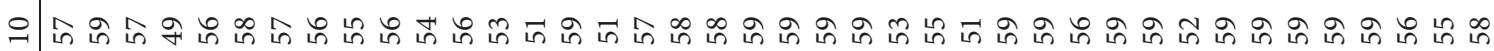

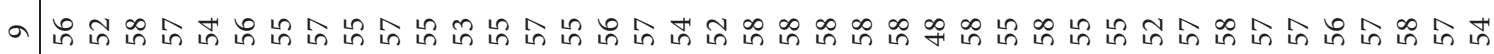

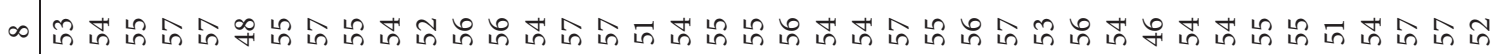

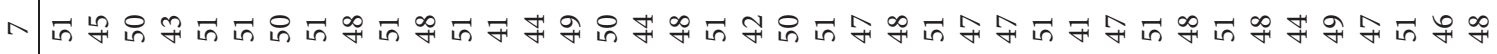

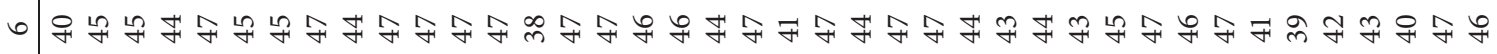
n

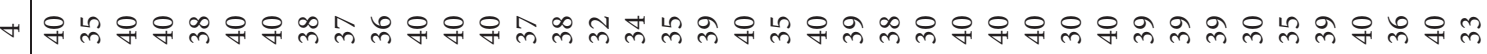
m 小 $-\vec{\sim} \stackrel{\infty}{\sim} \bumpeq 0 \div$

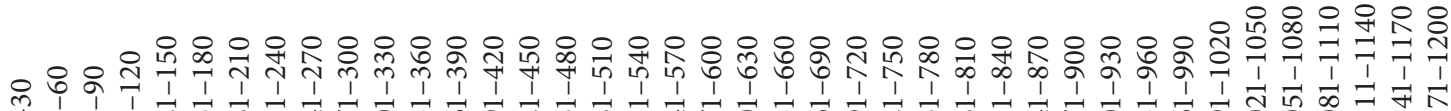

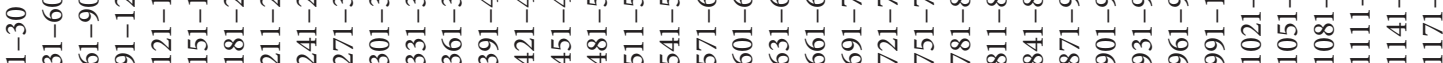

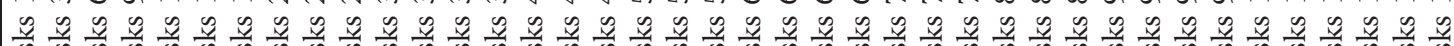

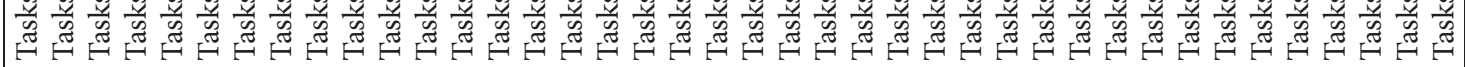




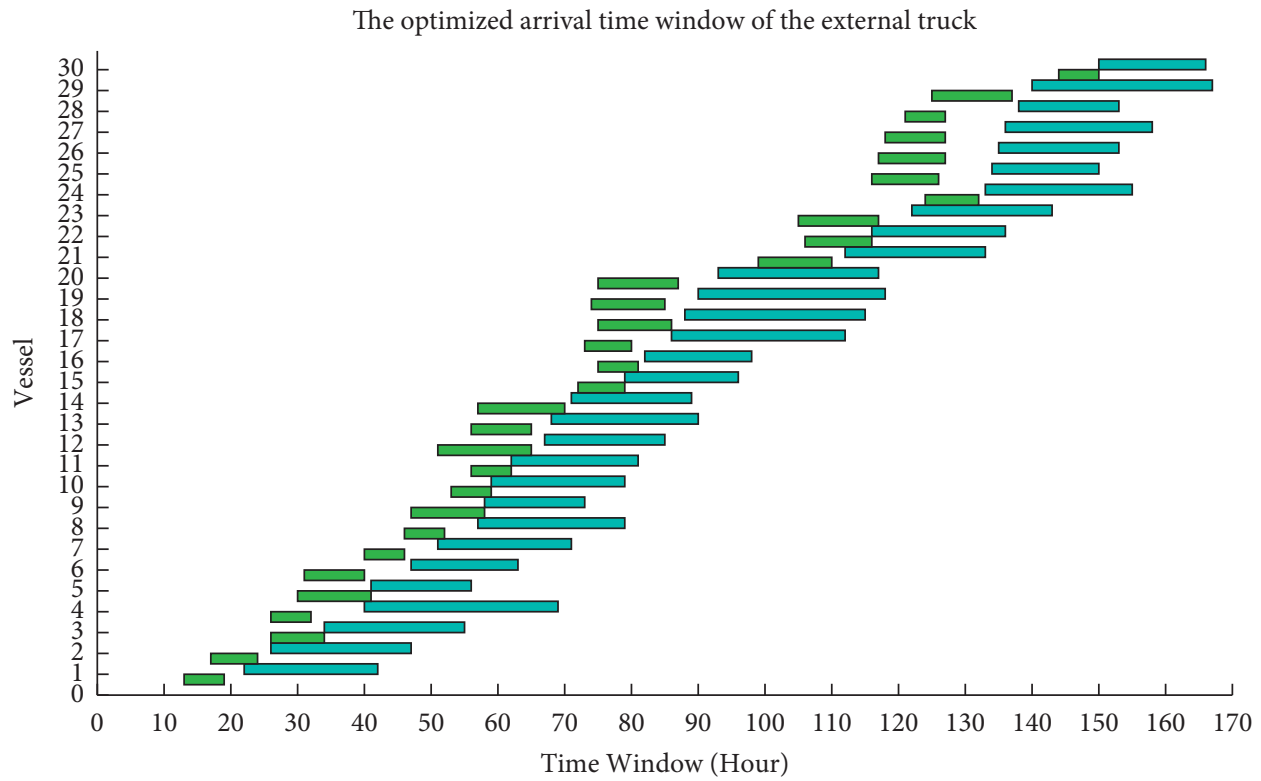

FIGURE 12: The optimized arrival time windows of the external trucks.

(2) The logistic chaotic sequence determines the mutation point

\section{Numerical Experiment and Result Analysis}

5.1. Case Description. The proposed model is applied to actual data prediction. The cycle is one week, including weekly vessel berthing plans, relevant container, and external trucks information. Figure 7 shows the operation process of the external trucks in the terminal. Taking 30 vessels docked within 7 days as an example, the total number of tasks is 1200; the arrival and departure time, terminal system data, and cost data of these vessels are collected to verify the proposed optimization model [8].

5.2. Parameter Value. Other input variables and input values as well as estimated times of arrival and departure of vessels are given in Tables 1 and 2, respectively.

5.3. Result Analysis. A simulation experiment was conducted in MATLAB 2018b to test the applicability of the adopted bilevel optimization model for the priority dispatch of emergency materials in terminals and the proposed the chaotic genetic algorithm based on logistic mapping (LCGA). The number of iterations was determined by attempting some pilot calculations. The L-CGA convergence is normally finalized at 60-70 generations; therefore, 200 iterations should be sufficient. The experimental results were as follows.

Figure 8 shows the convergence process of the population optimal values of the genetic algorithm, adaptive genetic algorithm, and chaotic genetic algorithm based on logistic mapping. The population optimal value of L-CGA reaches convergence in 70 generations. Figure 9 shows the comparison of the solution time of the three algorithms, and
Figure 10 shows the comparison of the solution results. According to the data, the cost of per container is 134.08 yuan/TEU in the Nanjing Port Longtan Container Co., Ltd.; in this study, the total cost is $1.97 E+06$, and the cost of per container is 82.06 yuan/TEU. It can be seen that the optimized chaotic genetic algorithm has faster solving speed and better results. In summary, L-CGA has a faster convergence rate, converges to the best solution, maintains population diversity, and enhances the global convergence of genetic algorithms. Therefore, L-CGA was chosen for solving the problem.

Tables 3 and 4 provide the task time window of the external trucks related to the vessel, where TA is the starting point of the time window, TB is the ending point of the time window, and the tables provide the specific operation time windows of all tasks.

Figure 11 shows the comparison of the truck arrivals in the real and optimized cases. The real solution generates a very high arrival peak in the middle of the week, which substantially exceeds the gate capacity. The result shows that using the optimized arrival patterns of external trucks can reduce the regularity of the truck arrivals and flatten the peaks of hourly truck arrivals.

Tables 5 and 6 provide the operating time window of the external trucks after considering the priority dispatch of emergency materials, which is the optimal plan for priority dispatch of emergency materials.

Figure 12 shows the optimized arrival time windows of the external trucks. The blue rectangles are the time windows before optimization, and the green parts are the time windows after optimization. It can be seen that the overlaps of the time windows are significantly reduced from 468 hours to 157 hours, a decrease of about $66.45 \%$. At the same time, the total task time windows have been reduced from 612 hours to 266 hours, a decrease of $56.54 \%$. 


\section{Conclusions}

This study investigated the priority dispatching of emergency materials at container terminals during an epidemic. The time window constraints were considered, and a bilevel optimization model for container terminals was built to ensure the priority dispatching of epidemic prevention materials. The irregular arrival of external trucks is a key factor affecting the congestion of terminal gates and yards and the low efficiency of the yard. Simultaneously, considering the serious problem of yard pressing during the epidemic, a penalty fee for insufficient yard space was added to the plan. This prevents the yard from having no space for storage when an external truck is delivered to the terminal, thereby increasing terminal congestion. The chaotic genetic algorithm based on logistic mapping was used to solve the bilevel optimization model. Experimental results showed that the bilevel optimization model proposed in this study and the chaotic genetic algorithm based on logistic mapping improve the speed and accuracy of the solution. The total cost of the external trucks in the terminal was reduced while ensuring the priority dispatch of emergency materials, reducing the overlapping part of the time window, and optimizing the arrival mode of external trucks, thereby reducing the waiting time for an external truck.

This study alleviates the congestion of container terminals and effectively ensures the supply of emergency medical supplies and living materials in an epidemic. Thus, the results of this study are of great significance to control the COVID-19 epidemic and treat infected patients. The limitation of this study is that it only considers the capacity constraints of the yard but does not consider the operating time window of the yard crane and does not involve the problem of yard crane task allocation. Therefore, in the future, we will consider other practical constraints in terminal operations, such as operation tasks and the time windows of quayside cranes and yard cranes. In addition, as all the vessels considered in this model have the same arriving periodicities, it will be more practical if multiple arriving patterns are considered in the future.

\section{Data Availability}

The data used to support the findings of this study are included within the article.

\section{Conflicts of Interest}

The authors declare that there are no conflicts of interest.

\section{Acknowledgments}

This work was supported by the National Social Science Foundation of China (18BGL109) and the authors would like to express their gratitude to it.

\section{References}

[1] Y. Yang, M. Zhong, Y. Dessouky, and O. Postolache, "An integrated scheduling method for AGV routing in automated container terminals," Computers \& Industrial Engineering, vol. 126, pp. 482-493, 2018.

[2] M. Ng, "Bounds on ship deployment in container shipping with time windows," Journal of the Operational Research Society, vol. 72, pp. 1252-1258, 2020.

[3] J. Nossack and E. Pesch, "A truck scheduling problem arising in intermodal container transportation," European Journal of Operational Research, vol. 230, no. 3, pp. 666-680, 2013.

[4] S. Shiri and N. Huynh, "Optimization of drayage operations with time-window constraints," International Journal of Production Economics, vol. 176, pp. 7-20, 2016.

[5] G. Chen, K. Govindan, and Z. Yang, "Managing truck arrivals with time windows to alleviate gate congestion at container terminals," International Journal of Production Economics, vol. 141, no. 1, pp. 179-188, 2013.

[6] M. Ma, H. Fan, X. Jiang, and Z. Guo, "Truck arrivals scheduling with vessel dependent time windows to reduce carbon emissions," Sustainability, vol. 11, no. 22, p. 6410, 2019.

[7] S. Yi, B. Scholz-Reiter, T. Kim, and K. H. Kim, "Scheduling appointments for container truck arrivals considering their effects on congestion," Flexible Services and Manufacturing Journal, vol. 31, no. 3, pp. 730-762, 2019.

[8] G. Chen and L. Jiang, "Managing customer arrivals with time windows: a case of truck arrivals at a congested container terminal," Annals of Operations Research, vol. 244, no. 2, pp. 349-365, 2016.

[9] G. Assadipour, G. Y. Ke, and M. Verma, "An analytical framework for integrated maritime terminal scheduling problems with time windows," Expert Systems with Applications, vol. 41, no. 16, pp. 7415-7424, 2014.

[10] J. P. Minas, N. C. Simpson, and Z. Y. Tacheva, "Modeling emergency response operations: a theory building survey," Computers \& Operations Research, vol. 119, Article ID 104921, 2020.

[11] P. Memari, R. Tavakkoli-Moghaddam, F. Navazi, and F. Jolai, "Air and ground ambulance location-allocation-routing problem for designing a temporary emergency management system after a disaster," Proceedings of the Institution of Mechanical Engineers, Part H: Journal of Engineering in Medicine, vol. 234, no. 8, pp. 812-828, 2020.

[12] C. Qi, J. Fang, and L. Sun, "Implementation of emergency logistics distribution decision support system based on GIS," Cluster Computing, vol. 22, no. 4, pp. 8859-8867, 2019.

[13] L. Lu and X. Luo, "Emergency transportation problem based on single-valued neutrosophic set," Discrete Dynamics in Nature and Society, vol. 2020, Article ID 4813497, 8 pages, 2020.

[14] F. Wang, Z. Pei, L. Dong, and J. Ma, "Emergency resource allocation for multi-period post-disaster using multi-objective cellular genetic algorithm," IEEE Access, vol. 8, pp. 8225582265, 2020.

[15] Y. Chen, Y. Wang, H. Wang, Z. Hu, and L. Hua, "Controlling urban traffic-one of the useful methods to ensure safety in Wuhan based on COVID-19 outbreak," Safety Science, vol. 131, Article ID 104938, 2020.

[16] Z. Liu, Z. Li, W. Chen, Y. Zhao, H. Yue, and Z. Wu, "Path optimization of medical waste transport routes in the emergent public health event of COVID-19: a hybrid optimization algorithm based on the immune-ant colony algorithm," International Journal of Environmental Research and Public Health, vol. 17, no. 16, 2020. 
[17] J. Pacheco and M. Laguna, "Vehicle routing for the urgent delivery of face shields during the COVID-19 pandemic," Journal of Heuristics, vol. 26, no. 5, pp. 619-635, 2020.

[18] H. Park, D. Son, B. Koo, and B. Jeong, "Waiting strategy for the vehicle routing problem with simultaneous pickup and delivery using genetic algorithm," Expert Systems with Applications, vol. 165, Article ID 113959, 2021.

[19] L. Escobar-Falcón, D. Álvarez-Martínez, J. Wilmer-Escobar, and M. Granada-Echeverri, "A specialized genetic algorithm for the fuel consumption heterogeneous fleet vehicle routing problem with bidimensional packing constraints," International Journal of Industrial Engineering Computations, vol. 12, no. 2, pp. 191-204, 2021.

[20] A. Mahpour, A. Nazifi, and A. M. Amiri, "Development of optimization model to reduce unloading and loading time at berth in container ports," Iranian Journal of Science and Technology, Transactions of Civil Engineering, vol. 45, 2021.

[21] Z. Avdagic, A. Smajevic, S. Omanovic, and I. Besic, "Path route layout design optimization using genetic algorithm: based on control mechanisms for on-line crossover intersection positions and bit targeted mutation," Journal of Ambient Intelligence and Humanized Computing, 2021.

[22] Y. Liu, W. Xu, Y. Chen, and H. Ma, "Chaos genetic algorithm with fitness selection adjustment strategy and its application," Computing Technology and Automation, vol. 38, no. 2, pp. 8-14, 2019. 